\title{
Bounding the inefficiency of outcomes in generalized second price auctions*
}

\author{
Ioannis Caragiannis ${ }^{\dagger}$ \\ Christos Kaklamanis ${ }^{\dagger}$ \\ Panagiotis Kanellopoulos ${ }^{\dagger}$ \\ Maria Kyropoulou ${ }^{\ddagger}$ \\ Brendan Lucier ${ }^{\S}$ \\ Renato Paes Leme \\ Éva Tardos $\|$
}

\begin{abstract}
The Generalized Second Price (GSP) auction is the primary auction used for monetizing the use of the Internet. It is well-known that truthtelling is not a dominant strategy in this auction and that inefficient equilibria can arise. Edelman et al. (AER, 2007) and Varian (IJIO, 2007) show that an efficient equilibrium always exists in the full information setting. Their results, however, do not extend to the case with uncertainty, where efficient equilibria might not exist.

In this paper we study the space of equilibria in GSP, and quantify the efficiency loss that can arise in equilibria under a wide range of sources of uncertainty, as well as in the full information setting. The traditional Bayesian game models uncertainty in the valuations (types) of the participants. The Generalized Second Price (GSP) auction gives rise to a further form of uncertainty: the selection of quality factors resulting in uncertainty about the behavior of the underlying ad allocation algorithm. The bounds we obtain apply to both forms of uncertainty, and are robust in the sense that they apply under various perturbations of the solution concept, extending to models with information asymmetries and bounded rationality in the form of learning strategies.

We present a constant bound (2.927) on the factor of the efficiency loss (price of anarchy) of the corresponding game for the Bayesian model of partial information about other participants and about ad quality factors. For the full information setting, we prove a surprisingly low upper bound of 1.282 on the price of anarchy over pure Nash equilibria, nearly matching a lower bound of 1.259 for the case of three advertisers. Further, we do not require that the system reaches equilibrium, and give similarly low bounds also on the quality degradation for any no-regret learning outcome. Our conclusion is that the number of advertisers in the auction has almost no impact on the price of anarchy, and that the efficiency of GSP is very robust with respect to the belief and rationality assumptions imposed on the participants.
\end{abstract}

JEL Classification: D44 - Auctions

Keywords: auction design, equilibrium analysis, price of anarchy, bayesian games, generalized second price auction, keyword auctions

\footnotetext{
* Preliminary versions of some of the results in this paper appeared in Paes Leme and Tardos, FOCS'10 [29], Lucier and Paes Leme, EC'11 [24], and Caragiannis, Kaklamanis, Kanellopoulos and Kyropoulou, EC'11 [8].

${ }^{\dagger}$ Computer Technology Institute and Press "Diophantus" \& Department of Computer Engineering and Informatics, University of Patras, Greece. Email: \{caragian, kakl,kanellop\}@ ceid.upatras.gr

${ }^{\ddagger}$ Department of Computer Science, University of Oxford, UK. Email: kyropoulecs . ox . ac.uk

${ }^{\S}$ Microsoft Research New England, USA. Email: brlucier@microsoft .com

"Google Research New York, USA. Email: renatoppl@google.com

"Corresponding author. Department of Computer Science, Cornell University, USA. Supported in part by NSF grants CCF0910940 and CCF-0729006, ONR grant N00014-98-1-0589, a Yahoo! Research Alliance Grant, and a Google Research Grant. Email: eva@cs.cornell.edu
} 


\section{Introduction}

The sale of advertising space on the Internet, or AdAuctions, is the primary source of revenue for many providers of online services. According to a recent report [13], $\$ 25.8$ billion dollars were spent in online advertisement in the US in 2010. The main part of this revenue comes from search advertisement, in which search engines display ads alongside organic search results. The success of this approach is due, in part, to the fact that providers can tailor advertisements to the intentions of individual users, which can be inferred from their search behavior. A search engine, for example, can choose to display ads that synergize well with a query being searched. However, such dynamic provision of content complicates the process of selling ad space to potential advertisers. Each search query generates a new set of advertising space to be sold, each with its own properties determining the applicability of different advertisements, and these ads must be placed near-instantaneously.

The now-standard mechanism for resolving online search advertisement requires that each advertiser places a bid that represents the maximum she would be willing to pay if a user clicked her ad. These bids are then resolved in an automated auction whenever ads are to be displayed. By far the most popular bidresolution method currently in use is the Generalized Second Price (GSP) auction, a generalization of the well-known Vickrey auction. In the GSP auction, there are multiple ad "slots" of varying appeal (e.g. slots at the top of the page are more effective). In two seminal papers Edelman et al. [11] and Varian [36] propose a simple model of the GSP auction that we will also adopt in this paper. They observe that truthtelling is not a dominant strategy under GSP, and GSP auctions do not generally guarantee the most efficient outcome (i.e., the outcome that maximizes social welfare). Nevertheless, the use of GSP auctions has been extremely successful in practice. This begs the question: are there theoretical properties of the Generalized Second Price auction that would explain its prevalence? Edelman et al. [11] and Varian [36] provide a partial answer to this question by showing that, in the full information setting, a GSP auction always has a Nash equilibrium that has same allocation and payments as the VCG mechanism. [11] and [36] give only informal arguments to justify the selection of envy-free equilibria.

We argue that the Generalized Second Price auction is best modeled as a Bayesian game of partial information. Modeling GSP as a full information game assumes that each auction is played repeatedly with the same group of advertisers, and during such repeated play the bids stabilize. The resulting stable set of bids is well modeled by a full information Nash equilibrium. The analyses of Edelman et al. [11] and Varian [36] provide important insight into the structure of the GSP auction under this assumption. However, the set and types of players can vary significantly between rounds of a GSP auction. Each query is unique, in the sense that it is defined not only by the set of keywords invoked but also by the time the query was performed, the location and history of the user, and many other factors. Search engines use complex machine learning algorithms to select the ads, and more importantly to determine appropriate quality scores (or factors) for each advertiser for a particular query, and then decide which advertiser to display. This results in uncertainty both about the competing advertisers, and about quality factors. We model this uncertainty by viewing the GSP auction as a Bayesian game, and ask: what are the theoretical properties of the Generalized Second Price auction taking into account the uncertainty that the advertisers face?

Bounding the quality of outcomes: Price of Anarchy. To answer the question above, we offer a quantitative understanding of the inefficiencies that can arise in GSP auctions, using a metric known as the Price of Anarchy. We show that the welfare generated by the auction in any equilibrium of bidding behavior is at least a $\frac{1}{\eta}$-fraction of the maximum achievable welfare (i.e., the welfare the auction could generate knowing the player types and quality factors in advance). The value of $\eta$ measures the robustness of an auction 
with respect to strategic behavior: in the worst case, how much can strategic manipulation harm the social welfare. The closer $\eta$ is to 1 , the more robust the auction is. An auction that always generates efficient outcomes at equilibrium would have price of anarchy equal to 1 . We bound the inefficiency of the outcomes both in the Bayesian version of the game as well as the full information game, and extend the analysis also for learning outcomes.

We develop a general technique for bounding the inefficiency of outcomes that allows us to do this in the most general setting, even in Bayesian games with multiple, correlated sources of uncertainty. Our framework of semi-smooth games is an extension of Roughgarden's [31] smoothness framework, that allows dealing with correlated distributions. Correlated distributions are an important feature of the GSP model, especially when modeling quality factors, as the same facts affect clickability and hence the quality factors for all advertisers. (For instance, an ad shown to a bot will not get a click independent of the advertiser.)

For mechanisms that are not dominant strategy truthful, like GSP auctions, price of anarchy analysis is a powerful tool for quantifying the potential loss of efficiency at equilibrium. We conduct this analysis both in a full information setting without uncertainty (in which the price of anarchy is surprisingly small, indicating a loss of at most $22 \%$ of the welfare), but also in a setting with uncertainty and a very general information structure, in which we prove that the price of anarchy is still bounded by a small constant. This shows that while the GSP auction is not guaranteed to be efficient, it is a reasonably good design, as remarkably, the welfare loss of these auctions is bounded by a value that does not depend on the number of players, the number of advertisements for sale, or the prior distributions on player types. In contrast, the variant of the Generalized Second Price auction that orders advertisers by their bid ignoring quality factors, which has been historically used by Yahoo!, results in a quality loss proportional to the range of quality factors, while randomly assigning advertisers to slots can result in a loss of efficiency proportional to the number of advertisers.

One feature of our results is that they hold for a variety of models regarding the rationality and the beliefs of the players. This robustness is particularly important in large-scale auctions conducted over the Internet, where assumptions of full information and/or perfect rationality of the participants are unreasonably strong.

The GSP auction and sources of uncertainty. By far the most popular auction method currently in use for search ads is the Generalized Second Price (GSP) auction, a generalization of the well-known Vickrey auction. The GSP auction is invoked every time a user queries a keyword of interest; it is a repeated auction in which players repeatedly bid for ad slots. However, modeling equilibrium strategies in a repeated game of this nature is notoriously difficult, and results in a game with a plethora of unnatural equilibria due to the possibility of bids representing threats for future rounds, optimal exploration of the bidding space, and so on. A common simplification used in the literature is to focus on auctions for a single keyword, and to suppose that players will quickly learn each others' valuations and reach a stationary equilibrium. Under this assumption, the stationary equilibrium would correspond naturally to a Nash equilibrium in the full information, one-shot version of the GSP auction [12]. It has therefore become common practice to study pure, full information equilibria of the one-shot game, as an approximation to expected behavior in the more general repeated game [11, 36, 28].

In reality, however, the set and types of players can vary significantly between rounds of a GSP auction: each query is unique, in the sense that it is defined not only by the set of keywords invoked but also the time the query was performed, the location and history of the user, and many other factors. This context is taken into account by an underlying ad allocation algorithm, which is controlled by the search engine. The ad allocation algorithm not only selects which advertisers will participate in an auction instance, but also assigns a quality factor to each advertiser. As a first approximation we can think of the quality factor as 
a score that measures how likely that participant's ad will be clicked for that query. These quality factors are then used to scale the bids of the advertisers. These scaled bids are known as effective bids, which can be viewed as bids derived from a similarly-modified effective type. Under our assumption that quality factors measure clickability, the effective type of an advertiser is the expected valuation of displaying the ad (valuation of the ad times its likelihood of getting a click). The effective bid and effective type of a player are therefore random variables, which can be thought of as the original valuations multiplied by quality scores computed exogenously by the search engine. Athey and Nekipelov [3] point out that the uncertainty in quality factors produces qualitative changes in the structure of the game. Thus, even if players converge to a stationary bidding pattern, the resulting equilibrium cannot be described as the outcome of a full information game.

We model the uncertainty about the effective types of advertisers as a Bayesian, partial information game. That is, the inherent uncertainty due to context and the ad allocation algorithm can be captured via prior distributions over effective types, even when the true types of all potential competitors are fully known. The appropriate equilibrium notion is then the Bayes-Nash equilibrium with respect to these distributions. Our model allows arbitrary correlations between the types and quality factors. The uncertainty of ad quality and allocation mostly comes from the query context, and hence is best modeled by correlated distributions of types and ad quality. Search engines use complex machine learning algorithms to compute quality factors based on all available information about the context, whose outcome is hard to predict for the advertisers. Search engines share distributional information about quality factors with advertisers. We model this by assuming that the advertisers are aware of the distribution of quality factors. Further, we also assume that the quality factors computed by the search engine correspond exactly to the clickability of the ad.

Summarizing, there are two main sources of uncertainty: the first is about the quality factors that the search engine attributes to each advertiser and the second is about the valuations (types) of the players. These sources are different in nature: each advertiser has knowledge of (and can condition her behavior on) her own type, whereas quality factors are fully exogenous and are only revealed ex post.

Asymmetric information. There are different types of players in advertising markets, which may have differing levels of information about their competitors. We assume all players know their own valuations correctly, but some smaller players (such as individual advertisers) might be clueless about the valuations of the other players and expected behavior of quality scores, while others (say bidding agencies or large companies with web advertising departments) may have a much better understanding of how individual rounds of the auction will proceed. Even among this latter group, different advertisers may have access to different information. We can model such information asymmetries by giving each player access to an arbitrary player-specific signal that can carry information about the effective types of the auction participants. Our bounds on social efficiency in the Bayesian model hold in settings with such asymmetry in information.

Learning players. So far we have considered equilibria of the auction game. Analyzing equilibria makes the strong assumption that players reach equilibrium play. Learning outcomes provide a very appealing generalization. A now standard model considers a repeated version of the game, and assumes that players employ strategies that give them vanishingly small regret over time. Roughly speaking, such a model assumes that players observe the bidding patterns of others and modify their own bids in such a way that their long-term performance is at least as good as a single optimal strategy chosen in hindsight. Notice that if all players employ the same (possibly randomized) strategy in each round, the resulting stable strategies form a Nash equilibrium. Therefore, the no-regret assumption of repeated play is a generalization of the notion of Nash equilibrium. Further, there are many simple bidding strategies that yield vanishing regret over time, as 
discussed below. The no-regret assumption does not require that players follow one of these algorithms; in fact, good play can result in better utility than simply no-regret, e.g., if the player can anticipate the behavior of other players. Rather, the assumption models a natural rationality: if there is a consistently good strategy players will attempt to learn this over time, and do at least as well (or better) as this good fixed strategy. In this sense, the no-regret assumption aims to capture the intuition that players attempt to learn beneficial bidding strategies over time, while also providing a generalization of Nash equilibrium play. We view the existence of simple learning algorithms as supporting this assumption. If all players have no-regret this will cause the empirical distribution of the bids to converge to a coarse correlated equilibrium of the game, a slight generalization of the well-known correlated equilibrium.

We therefore assume that the players use algorithms to learn how to best bid given their valuation and signal, and achieve vanishing regret over time. In other words, for each possible valuation and signal, repeated auctions allow players to learn how to best bid taking into account the varying bids of other players, and the uncertainty about quality factors, other players' valuations, and bidding strategies. We will consider the quality degradation of the average social outcome when all players employ strategies with small regret. Blum et al. [6] introduced the term Price of Total Anarchy for this analog of the price of anarchy.

Approximate rationality. One of the fundamental assumptions in auction analysis is that all players are perfectly rational utility optimizers. However, in reality (and especially in large online settings), it is natural to assume that some fraction of the players participating in an advertising auction might have unsophisticated bidding strategies. In fact, some players may not even play at equilibrium in the single-shot approximation of the GSP auction, or may only be able to find strategies that are approximately utility-maximizing. We discuss the robustness of our bounds to the presence of players bidding with limited (or no) rationality. As we shall see, the GSP auction has the property that its social welfare guarantees degrade continuously when our assumptions about the rationality of the players are relaxed.

\subsection{Our results}

We present the following results.

- Our main result is a bound on the Bayesian price of anarchy for the GSP auction. Specifically, we show that the price of anarchy is at most 2.927, meaning that the social welfare in any Bayes-Nash equilibrium is at least $1 / 2.927$ of the optimal social welfare. Notice that this is an unconditional bound, as we make no assumptions on the distribution on valuation profiles and quality factors (it can, for example, be correlated) or on the number of players or slots. In the main part of the paper, we prove weaker bounds for both the full information and the Bayesian game, and only sketch the stronger bounds. We believe that the weaker bounds are interesting in their own right, and show the main techniques of the paper in a way that is easier to read. We defer the details of the stronger bounds to the Appendix.

Perhaps just as important as the bound, however, is the straightforward and robust nature of the GSP auction. In particular, our results extend to provide the same welfare guarantees for outcomes of no-regret learning: the average social welfare when players play repeatedly in order to minimize total regret, in a Bayesian setting, is within a $1 / 2.927$ factor of the optimal social welfare. In fact, some of our bounds for learning outcomes require only that the players have no regret for a particular natural strategy of shading their bids. The bounds continue to hold even if players have asymmetric access to distributional information, in the form of exogenously provided signals. It also degrades continuously in the presence of approximately rational players or a small fraction of irrational players 
as explained in Appendix $\mathrm{D}$. The results also extend to the case when possible bid space is discretized (i.e., players need to bid in integer number of pennies). This case is interesting both as in practice bids do come from such a discrete space, and also as in the discrete case, the existence of Nash equilibria is guaranteed. In fact, using a result of Athey [2] and Reny [30], in the discrete case if player types and quality factors are drawn independently, one can also show that the existence of pure strategy equilibria that are monotone in the types is guaranteed.

- We achieve the bounds on the solution quality by identifying a property that encapsulates some of the insight. Roughgarden [31] identified a class of games that he termed smooth games, defined via a similar property that is used to bound the price of anarchy. We identify a stronger property, semismoothness, that is satisfied by the GSP auction, and is strong enough to also imply price of anarchy bounds even in the Bayesian setting with arbitrarily correlated types.

- We provide improved results for the case where there is no uncertainty, which is the traditional setting studied in [11, 36]. If valuations and quality factors are fixed, we prove that the social welfare in any pure Nash equilibrium is within a factor of 1.282 of the optimal one and show that this bound is essentially tight by providing a lower bound of 1.259 . Also, we show a bound of 2.310 for coarse correlated equilibria; as discussed above, this implies the same bound on the social welfare for learning outcomes when players with fixed (effective) types minimize their regret in a repeated auction. This bound of 2.310 holds for mixed Nash equilibria as well.

\subsection{Related work}

Due to their central role in Internet monetization, sponsored search auctions have received considerable attention in the past years. From the optimization perspective, they were first considered by Mehta et al. [26]. A classical game-theoretical modeling of sponsored search auctions was proposed simultaneously by Edelman et al. [11] and Varian [36]. See the surveys of Lahaie et al. [20] and Maille et al. [25] for an overview of subsequent developments.

The model we adopt follows [11, 36]. In those two seminal papers, the authors notice that even though truthtelling is not a dominant strategy under GSP, the full information game always has a Nash equilibrium that has same allocation and payments as the VCG mechanism. They focus on a subclass of Nash equilibria which is called envy-free equilibria in [11] and symmetric equilibria in [36]. They show that such equilibria always exist and are always efficient. In this class, an advertiser would not be better off after switching bids with the advertiser just above her. Note that this is a stronger requirement than in Nash equilibria, which are defined considering only unilateral deviations by the advertisers, and if an advertiser unilaterally switches to a slot with higher click-through-rate, she pays more than the advertiser at that slot paid. In [11, 12, 36], informal arguments are presented to justify the selection of envy-free equilibria, but no formal game-theoretical analysis is done. We believe it is an important question to go beyond this and prove efficiency guarantees for all Nash equilibria. Lahaie [19] also considers the problem of bounding the social welfare obtained at equilibrium, but restricts attention to the special case that click-through-rates decay exponentially along the slots with a factor of $\frac{1}{\delta}$. Under this assumption, Lahaie proves a price of anarchy of $\min \left\{\frac{1}{\delta}, 1-\frac{1}{\delta}\right\}$.

Gomes and Sweeney [16] study the GSP auction in the Bayesian setting, where player types are drawn from independent and identical distributions (without considering the uncertainty due to quality factors). They show that, unlike the full information case, there may not exist symmetric or socially optimal equilibria in this model, and obtain sufficient conditions on click-through-rates that guarantee the existence of 
a symmetric and efficient equilibrium. Athey and Nekipelov [3] study the effect of uncertainty of quality factors both from a theoretical and an empirical perspective.

The study of price of anarchy for non-truthful auction mechanisms (especially in the Bayesian setting) was initiated by Christodoulou et al. [9] and developed in Lucier and Borodin [23], Lucier [22], and most recently in the work of Bhawalkar and Roughgarden [5]. To the best of our knowledge, the current paper is the first one in which the price of anarchy bounds hold when player valuations are drawn from a correlated distribution. In truthful mechanism design, the study of correlated valuations has a long history - see Cremer and McLean [10] for an early reference.

The study of regret-minimization goes back to the work of Hannan on repeated two-player games [17]. Since then, a number of simple algorithms (to be thought of as adaptive procedures) that guarantee no-regret have been proposed in the literature. Initial work in this area focused on the stronger requirement of finding simple adaptive procedures through which the play converges to the set of correlated equilibria, requiring that players have a stronger form of no-regret that is called no internal regret (see the survey by Blum and Mansour [7] for a discussion of such procedures and a comparison). Foster and Vohra [14] obtained such a procedure, and Fudenberg and Levine [15] presented a different one. Hart and Mas-Collel's regret matching strategy [18] or the multiplicative weight updating strategy of [21] (see also [1]) are two procedures that become especially simple when used to guarantee only no-regret (as opposed to no internal regret). These classical learning algorithms assume that players learn outcomes and strategies of all participants in each round, but have also been extended to situations where in each round, a player observes only her own outcome, or even realizations of her outcome in case it is randomized. We refer to Auer et al. [4] for a detailed discussion on this matter.

Adaptive procedures that guarantee no-regret define a play that converges to the set of coarse correlated equilibria. Blum et al. [6] apply regret-minimization to the study of inefficiency in repeated games, coining the term "price of total anarchy" for the worst-case ratio between the optimal objective value and the average objective value when players minimize regret.

Roughgarden [31] identifies a class of games that he terms smooth games where the price of anarchy and price of total anarchy are identical. See also [27] and [33], for subsequent refinements. Since the initial conference versions of our Bayesian bound of [24] and [8], Roughgarden [32] and independently Syrgkanis [34] show that the bounds proved via smoothness also extend to the Bayesian price of anarchy assuming a variant of the smoothness assumption (called universal smoothness in [34]) if player types are drawn from independent distributions. See [35] for such an extension theorem without the stronger assumption. In this paper we isolate a stronger property related to smoothness that encapsulates many of the insights that drive our bounds and allows us to extend our bounds for the Bayesian price of anarchy with correlated distributions.

Some of the results in this paper appeared in preliminary conference versions. Paes Leme and Tardos [29] study equilibria of GSP auctions and give upper bounds on the price of anarchy in pure, mixed, and Bayesian strategies; achieving bounds of 1.618, 4, and 8, respectively. Lucier and Paes Leme [24] and Caragiannis et al. [8] improve these bounds to 3.16 and 3.037 respectively for Bayesian games, and 1.282 and 2.31 for pure Nash and learning outcomes for full information games (as well as mixed Nash equilibria), and extend them to apply to equilibria with correlated valuations and learning outcomes. Here we further improve the bounds, present and also improve the proofs, and extend the results to games with uncertainty about quality factors in addition to player types. 


\section{Model and Equilibrium Concepts}

We consider an auction with $n$ advertisers and $n$ slots 1 . Each advertiser $i$ has a private type $v_{i}$, representing her valuation per click received. The sequence $\mathbf{v}=\left(v_{1}, \ldots, v_{n}\right)$ is referred to as the type profile (or valuation profile). We will write $\mathbf{v}_{-i}$ for $\mathbf{v}$ excluding the $i$ th entry, so that $\mathbf{v}=\left(v_{i}, \mathbf{v}_{-i}\right)$.

An outcome is an assignment of advertisers to slots. An outcome can be viewed as a permutation $\pi$ with $\pi(k)$ being the advertiser assigned to slot $k$. The probability of a click depends on the slot as well as the advertiser shown in the slot. We use the model of separable click probabilities. We assume slots have associated click-through-rates $\alpha_{1} \geq \alpha_{2} \geq \ldots \geq \alpha_{n}$, and each advertiser $i$ has a quality factor $\gamma_{i}$ that reflects the clickability of the ad. When advertiser $i$ is assigned to the $k$-th slot, she gets $\alpha_{k} \gamma_{i}$ clicks.

A mechanism for this auction elicits a bid $b_{i} \in \mathbb{R}_{+}:=[0, \infty)$ from each advertiser $i$, which is interpreted as a type declaration, and returns an assignment as well as a price $p_{i}$ per click for each advertiser. If advertiser $i$ is assigned to slot $j$ at a price of $p_{i}$, her utility is $\alpha_{j} \gamma_{i}\left(v_{i}-p_{i}\right)$, which is the number of clicks received times profit per click. The social welfare of outcome $\pi$ is $S W(\pi, \mathbf{v}, \gamma)=\sum_{j} \alpha_{j} \gamma_{\pi(j)} v_{\pi(j)}$, the total value of the solution for the participants. The social welfare also depends on the click-through-rates $\alpha_{j}$, but throughout the paper we will assume they are fixed and common knowledge, and as a result we suppress them in the notation. The optimal social welfare is $\operatorname{OPT}(\mathbf{v}, \gamma)=\max _{\pi} S W(\pi, \mathbf{v}, \gamma)$, the welfare generated by the socially efficient outcome. Note that the efficient outcome sorts advertisers by their effective values $\gamma_{i} v_{i}$, and assigns them to slots in this order. The effective value can be thought of as the expected value of showing the ad in a slot with click-through-rate equal to 1 .

We focus on a particular mechanism, the Generalized Second Price auction, which works as follows. Given bid profile $\mathbf{b}$, we define the effective bid of advertiser $i$ to be $\gamma_{i} b_{i}$, which is her bid modified by her quality factor, analogous to the effective value defined above. The auction sets $\pi(k)$ to be the advertiser with the $k$ th highest effective bid (breaking ties arbitrarily). That is, the GSP mechanism assigns slots with higher click-through-rate to advertisers with higher effective bids. Payments are then set according to critical value: the smallest bid that guarantees the advertiser the same slot. When advertiser $i$ is assigned to slot $k$ (that is, when $\pi(k)=i$ ), this critical value is defined as

$$
p_{i}=\frac{\gamma_{\pi(k+1)}}{\gamma_{i}} b_{\pi(k+1)}
$$

where we take $b_{n+1}=0$. We will write $u_{i}(\mathbf{b}, \gamma)$ for the utility derived by advertiser $i$ from the GSP mechanism when advertisers bid according to $\mathbf{b}$ :

$$
u_{i}(\mathbf{b}, \gamma)=\alpha_{\pi^{-1}(i)} \gamma_{i}\left(v_{i}-p_{i}\right)=\alpha_{\pi^{-1}(i)}\left[\gamma_{i} v_{i}-\gamma_{\pi_{\left(\pi^{-1}(i)+1\right)}} b_{\pi\left(\pi^{-1}(i)+1\right)}\right] .
$$

Notice that $\pi$ is a function of $\mathbf{b}, \gamma$ as well. In places where we need to be more explicit, we will write $\pi(\mathbf{b}, \gamma, j)$ to be the advertiser assigned to slot $j$ by GSP when quality factors are $\gamma$ and the advertisers bid according to $\mathbf{b}$. We will also write $\sigma(\mathbf{b}, \gamma, i)$ for the slot assigned to advertiser $i$, again when advertisers bid according to $\mathbf{b}$ and quality factors are $\gamma$. In other words, $\sigma(\mathbf{b}, \gamma, \cdot)=\pi^{-1}(\mathbf{b}, \gamma, \cdot)$. We write $\pi^{i}\left(\mathbf{b}_{-i}, \gamma, j\right)$ to be the advertiser that would be assigned to slot $j$ if advertiser $i$ did not participate in the auction. When $\mathbf{b}$ and $\gamma$ are clear from the context, we write $\pi(i)$ and $\sigma(i)$ instead of $\pi(\mathbf{b}, \gamma, i)$ and $\sigma(\mathbf{b}, \gamma, i)$. We will also write $\nu(\mathbf{v}, \gamma)$ for the optimal assignment of slots to advertisers for valuation profile $\mathbf{v}$, so that $\nu(\mathbf{v}, \gamma, i)$ is the slot that would be allocated to advertiser $i$ in the optimal assignment 2 .

\footnotetext{
${ }^{1}$ We note that we can handle unequal numbers of slots and advertisers by adding virtual slots with click-through-rate zero or virtual advertisers with zero valuation per click.

${ }^{2}$ We note that, since GSP makes the optimal assignment for a given bid declaration, we actually have that $\nu(\mathbf{v}, \gamma, i)$ and $\sigma(\mathbf{v}, \gamma, i)$ are identically equal. We define $\nu$ mainly for use when emphasizing the distinction between an efficient assignment for a valuation profile and the assignment that results from a given bid profile.
} 
We will consider rational behavior under various models of the information available to the advertisers. In general, the advertisers are engaged as players in a game defined by the auction mechanism; each of them aims to select a bidding strategy that maximizes her utility. In the following, we use the terms advertiser and player interchangeably. We group our models into full information and partial information ones. In all models we assume that the values $\alpha_{j}$ are fixed and commonly known to all players. In our full information settings, we assume that the quality factors $\gamma_{i}$ as well as the valuation profile $\mathbf{v}$ are also common knowledge. In our Bayesian setting of partial information, we assume that the profile of quality factors is unknown to all players, and the type $v_{i}$ is private knowledge known only to player $i$, but they are randomly drawn from a commonly known joint distribution $(\mathbf{F}, \mathbf{G})$ of quality factors and valuation profiles. It will turn out that bidding more than one's true type (overbidding) is a dominated strategy in the mechanism we consider. So, we will focus on non-overbidding (or conservative) players; see Section 2.3 for a discussion.

\subsection{Full information setting}

In the full information setting, the valuation profile $\mathbf{v}$ and quality factors $\gamma_{i}$ are fixed and common knowledge. We will therefore tend to drop dependencies on $\gamma$ from our notation when working in the full information setting. In this setting, a pure strategy for player $i$ is a bid $b_{i} \in \mathbb{R}_{+}$. We say that the bid profile $\mathbf{b}$ is a (pure) Nash equilibrium if there is no deviation from which the player can profit, i.e., for all $b_{i}^{\prime} \in \mathbb{R}_{+}$,

$$
u_{i}\left(b_{i}, \mathbf{b}_{-i}\right) \geq u_{i}\left(b_{i}^{\prime}, \mathbf{b}_{-i}\right) .
$$

It is known that a pure Nash equilibrium always exists in this setting [11, 36]. We can therefore define the (pure) Price of Anarchy to be

$$
\sup _{\mathbf{v}, \mathbf{b} \in N E} \frac{O P T(\mathbf{v})}{S W(\pi(\mathbf{b}), \mathbf{v})}
$$

where NE is the set of pure Nash equilibria (assuming no overbidding; see Section 2.3).

Similarly, a mixed strategy is a randomized bid $b_{i}$, which is a distribution over possible bids. A mixed Nash equilibrium is a profile of bid distributions $\mathbf{b}$ such that for all $i$ and all alternative strategies $b_{i}^{\prime}$,

$$
\left.\left.\mathbb{E}_{\mathbf{b}}\left[u_{i}\left(b_{i}, \mathbf{b}_{-i}\right)\right)\right] \geq \mathbb{E}_{\mathbf{b}}\left[u_{i}\left(b_{i}^{\prime}, \mathbf{b}_{-i}\right)\right)\right]
$$

Note that, unlike more general solution concepts we will discuss in a while, the bid distributions of different players at a mixed Nash equilibrium are independent. We define the (mixed) Price of Anarchy to be the worst-case ratio between optimal social welfare and expected social welfare in GSP across all valuation profiles and all mixed Nash equilibria:

$$
\sup _{\mathbf{v}, \mathbf{b} \in N E} \frac{O P T(\mathbf{v})}{\mathbb{E}_{\mathbf{b}}[S W(\pi(\mathbf{b}), \mathbf{v})]}
$$

\subsection{Bayesian setting}

In the Bayesian setting of partial information, we suppose that the valuation profile and the quality factors are drawn from a publicly known (possibly correlated) joint distribution $(\mathbf{F}, \mathbf{G})$. A strategy for player $i$ is a (possibly randomized) mapping $b_{i}: \mathbb{R}_{+} \rightarrow \mathbb{R}_{+}$, mapping her type $v_{i}$ to a bid $b_{i}\left(v_{i}\right)$. Notice that a player is not able to condition her bid on the quality factors, since they are only known to the search engine, and not to the advertisers. 
We write $\mathbf{b}(\mathbf{v})=\left(b_{1}\left(v_{1}\right), \ldots, b_{n}\left(v_{n}\right)\right)$ to denote the profile of bids that results when $\mathbf{b}$ is applied to type profile $\mathbf{v}$. We then say that strategy profile $\mathbf{b}$ is a Bayes-Nash equilibrium for distributions $\mathbf{F}, \mathbf{G}$ if, for all $i$, all $v_{i}$, and all alternative strategies $b_{i}^{\prime}$,

$$
\mathbb{E}_{\mathbf{v}_{-i}, \gamma, \mathbf{b}}\left[u_{i}\left(b_{i}\left(v_{i}\right), \mathbf{b}_{-i}\left(\mathbf{v}_{-i}\right), \gamma\right) \mid v_{i}\right] \geq \mathbb{E}_{\mathbf{v}_{-i}, \gamma, \mathbf{b}}\left[u_{i}\left(b_{i}^{\prime}\left(v_{i}\right), \mathbf{b}_{-i}\left(\mathbf{v}_{-i}\right), \gamma\right) \mid v_{i}\right] .
$$

That is, each player maximizes her expected utility by bidding in accordance with strategy $b_{i}(\cdot)$, assuming that the other players bid in accordance with strategies $\mathbf{b}_{-i}(\cdot)$, where expectation is taken over the distribution of the other players' types conditioned on $v_{i}$, any randomness in their strategies, and the quality factors. We define the Bayes-Nash Price of Anarchy to be

$$
\sup _{\mathbf{F}, \mathbf{G}, \mathbf{b}(\cdot) \in B N E} \frac{\mathbb{E}_{\mathbf{v}, \gamma}[O P T(\mathbf{v}, \gamma)]}{\mathbb{E}_{\mathbf{v}, \gamma, \mathbf{b}(\mathbf{v})}[S W(\pi(\mathbf{b}(\mathbf{v}), \gamma), \mathbf{v}, \gamma)]}
$$

where BNE is the set of all Bayes-Nash equilibria (again assuming no overbidding; see below).

\subsection{No overbidding}

It is important to note that, in both the full information and Bayesian settings, any bid $b_{i}>v_{i}$ is dominated by the bid $b_{i}=v_{i}$ in the GSP auction. If by bidding $b_{i}>v_{i}$, the next highest effective bid is greater than $\gamma_{i} v_{i}$, then the player gets negative utility. If on the other hand, the next highest effective bid is smaller or equal than $\gamma_{i} v_{i}$, then bidding $b_{i}=v_{i}$ would get the same slot and payment. Based on this, we make the following assumption for the rest of the paper:

Assumption: Players are conservative and do not employ overbidding strategies in GSP auctions. This means that for pure strategies $b_{i} \leq v_{i}$, for mixed strategies $\mathbb{P}\left(b_{i}>v_{i}\right)=0$, and for Bayesian strategies $\mathbb{P}\left(b_{i}\left(v_{i}\right)>v_{i}\right)=0$ for all $v_{i}$.

We use this assumption to rule out unnatural equilibria in which advertisers apply certain dominated strategies. We remark that, in these equilibria, the social welfare may be arbitrarily worse than the optimal. It is therefore necessary to exclude such dominated strategies in order to obtain meaningful bounds on the price of anarchy. We note, however, that this phenomenon is not specific to the GSP auction: such degenerate equilibria exist even in the Vickrey auction for a single good, where truthful bidding is a weakly dominant strategy. Since the Vickrey auction is a special case of GSP auctions (where one slot has $\alpha_{1}=1$, all other slots have $\alpha_{i}=0$ and all quality factors have $\gamma_{i}=1$ ), this issue carries over to our setting. Consider the example of a single-item Vickrey auction, where truthful bidding of $b_{i}=v_{i}$ is a weakly dominant strategy. Yet with overbidding, there are equilibria where an arbitrary player bids excessively high (and hence wins), while everyone else bids 0 . If the player bidding high has a low valuation, this results in a high price of anarchy. Note, however, that this Nash equilibrium seems very artificial as it depends crucially on the low valuation player using the dominated strategy of overbidding. Indeed, such an advertiser is exposed to the risk of negative utility (if some other advertiser submits a new bid between her valuation and bid) without any benefit. We therefore take the position that advertisers will avoid such dominated strategies when participating in the GSP auction.

\subsection{Signals and information asymmetry}

We define an extension of the setting above, incorporating a Bayesian version of information asymmetry. In this model, each player's type consists of a signal $s_{i}$ drawn from an arbitrary signal space $S$. The signal of 
player $i$ includes her valuation $v_{i}\left(s_{i}\right)$ and can contain other privately-gained insight that refines the player $i$ 's conditional distribution over the space of other players' types and quality factors. The signals and quality factors come from a publicly known joint distribution $\left(\mathbf{F}^{\prime}, \mathbf{G}\right)$, which can be arbitrarily correlated.

In this model, a strategy is a bidding function that maps $s_{i}$, a signal, to a distribution of possible bids. The bid profile $\mathbf{b}$ is a Bayes-Nash equilibrium in the asymmetric information model if, for all $i$ and all alternative bidding functions $b_{i}{ }^{\prime}$,

$$
\mathbb{E}_{\mathbf{s}_{-i}, \gamma, \mathbf{b}}\left[u_{i}\left(b_{i}\left(s_{i}\right), \mathbf{b}_{-i}\left(\mathbf{s}_{-i}\right), \gamma\right) \mid s_{i}\right] \geq \mathbb{E}_{\mathbf{s}_{-i}, \gamma, \mathbf{b}}\left[u_{i}\left(b_{i}^{\prime}\left(s_{i}\right), \mathbf{b}_{-i}\left(\mathbf{s}_{-i}\right), \gamma\right) \mid s_{i}\right]
$$

In this model, the Price of Anarchy is defined as

$$
\sup _{\mathbf{F}^{\prime}, \mathbf{G}, \mathbf{b}(\cdot) \in B N E} \frac{\mathbb{E}_{\mathbf{s}, \gamma}[O P T(\mathbf{v}(\mathbf{s}), \gamma)]}{\mathbb{E}_{\mathbf{s}, \gamma, \mathbf{b}(\mathbf{s})}[S W(\pi(\mathbf{b}(\mathbf{s}), \gamma), \mathbf{v}(\mathbf{s}), \gamma)]}
$$

where BNE is the set of Bayes-Nash equilibria with respect to distribution $\mathbf{F}^{\prime}$ over signals, with no overbidding.

The presence of signals captures the notion that some advertisers might have a better potential to infer the other advertisers' valuations than others, or may be endowed with privileged information. We do note, however, that players do know their own valuations $v_{i}\left(s_{i}\right)$, and also are aware of the profile of bidding strategies $\mathbf{b}(\cdot)$ and the distribution $\mathbf{F}^{\prime}$, so that players can rationalize about the effects of signals upon the bidding behavior of their opponents.

\subsection{Repeated auctions and regret minimization}

We now consider the GSP auction in a repeated-game setting. In this model, the GSP auction is run $T \geq 1$ times. We will distinguish between two variants of this model: the full information model and the model with uncertainty.

Full information model. Each round of the GSP auction occurs with the same slots and players. The valuation profile $\mathbf{v}$ of the players and the quality factors do not change between rounds, but the players are free to change their bids. We write $b_{i}^{t}$ for the bid of player $i$ on round $t$. We refer to a $D=\left(\mathbf{b}^{1}, \ldots, \mathbf{b}^{T}, \ldots\right)$ as an (infinite) declaration sequence. Given declaration sequence $D$, we will write $D^{T}$ to mean the prefix of $D$ of length $T$; that is, $D^{T}=\left(\mathbf{b}^{1}, \ldots, \mathbf{b}^{T}\right)$. Given a (finite or infinite) declaration sequence $D$, we will write $\pi(D)$ for the sequence of permutations generated by GSP on input $D$. The average social welfare generated by GSP on a finite input sequence $D^{T}$ of length $T$ is $S W\left(\pi\left(D^{T}\right), \mathbf{v}\right)=\frac{1}{T} \sum_{t=1}^{T} S W\left(\pi\left(\mathbf{b}^{t}\right)\right.$, v). The average social welfare generated by GSP on an infinite input sequence $D$ is then defined to be $S W(\pi(D), \mathbf{v})=$ $\liminf _{T \rightarrow \infty} S W\left(\pi\left(D^{T}\right), \mathbf{v}\right)$.

The full range of equilibria in such a repeated game is very rich, so we restrict ourselves to a particular non-equilibrium form of play that nevertheless captures the intuition that players learn appropriate bidding strategies over time, without necessitating convergence to a stationary equilibrium.

We say that declaration sequence $D=\left(\mathbf{b}^{1}, \ldots, \mathbf{b}^{T}, \ldots\right)$ minimizes external regret for player $i$ if, for any fixed declaration $b_{i}^{\prime}$,

$$
\sum_{t \leq T} u_{i}\left(b_{i}^{t}, \mathbf{b}_{-i}^{t}\right) \geq \sum_{t \leq T} u_{i}\left(b_{i}^{\prime}, \mathbf{b}_{-i}^{t}\right)+R(T)
$$

where $R(T) / T \rightarrow 0$ as $T$ grows large. That is, as $T$ grows large, the utility of player $i$ in the limit is no worse than the utility of the optimal fixed strategy in hindsight. The Price of Total Anarchy [6] is the 
worst-case ratio between optimal social welfare and the average social welfare obtained by GSP across all declaration sequences that minimize external regret for all players. That is, the price of total anarchy is

$$
\sup _{\mathbf{v}, D} \frac{O P T(\mathbf{v})}{S W(\pi(D), \mathbf{v})}
$$

where the supremum is taken over (infinite) declaration sequences that minimize external regret for all players.

To this point we have not discussed how the players achieve vanishing regret, and indeed our results are agnostic to this process. There are many known learning algorithms that guarantee vanishing regret as $T$ goes to infinity. These algorithms require only that a player observes her realized payoff after each round. That is, to facilitate learning it is enough if players see whether or not their ad was clicked and, if so, the price of the click. In particular, they do not need to learn outcomes or bids of other players, nor even the actual slot their ad was placed in. For an extensive discussion on no-regret algorithms with limited feedback, we refer to Auer et al. [4]. Further, it is known that the price of total anarchy is closely related to an equilibrium notion for the single-shot game known as coarse correlated equilibrium. We discuss this relationship further in Section 5.1

Learning with uncertainty. Next we describe our model of learning in repeated GSP auctions with uncertainty. In this model, each round of the GSP auction occurs with the same slots, but the valuation profile $\mathbf{v}$ and quality factors $\gamma$ are redrawn from $(\mathbf{F}, \mathbf{G})$ on each round 3 . These changes to ad quality and types can be thought of as being due to the context of the search query that initiates each auction instance, which can change between rounds. As before, learning requires only that players observe their own outcome each round, and not the results for other players. I.e., a player learns whether or not her ad was clicked, and if so the price per click, but does not necessarily observe the outcomes or bids of other players nor the realization of $\gamma$. We again refer to Auer et al. [4] for a discussion of no-regret algorithms with limited feedback.

Suppose that each player has a finite type space 4 . Let $\mathbf{v}^{t}, \gamma^{t}$ be the type profile and quality factors drawn at round $t$. Given a declaration sequence $D$ and type $\tilde{v}_{i}$ for player $i$, we denote by $I\left(i, \tilde{v}_{i}\right)$ the subsequence of $D$ consisting of the set of rounds in which player $i$ has type $\tilde{v}_{i}$, i.e., $I\left(i, \tilde{v}_{i}\right)=\left\{t ; v_{i}^{t}=\tilde{v}_{i}\right\}$. Define $I^{T}\left(i, \tilde{v}_{i}\right)$ analogously with respect to $D^{T}$. Given a sequence of type profiles and quality factors that represent the realization of these random quantities over the rounds of the auction, we say that player $i$ has vanishing regret in declaration sequence $D$ if player $i$ has vanishing regret (in the sense of the full information game) on the subsequence $I\left(i, \tilde{v}_{i}\right)$ of $D$ for each possible type $\tilde{v}_{i}$. Formally:

$$
\sum_{t \in I^{T}\left(i, \tilde{v}_{i}\right)} u_{i}\left(b_{i}^{t}, \mathbf{b}_{-i}^{t}, \gamma^{t}\right) \geq \sum_{t \in I^{T}\left(i, \tilde{v}_{i}\right)} u_{i}\left(b_{i}^{\prime}, \mathbf{b}_{-i}^{t}, \gamma^{t}\right)+R\left(\left|I^{T}\left(i, \tilde{v}_{i}\right)\right|\right)
$$

for $R(T) / T \rightarrow 0$ as $T \rightarrow \infty$. Notice that since $\mathbf{v}^{t}$ is independently and identically distributed in each round, we have $\left|I^{T}\left(i, \tilde{v}_{i}\right)\right| \rightarrow \infty$ as $T \rightarrow \infty$. Now, we can define the Price of Total Anarchy with uncertainty as:

$$
\sup _{\left\{\mathbf{v}^{t}\right\},\left\{\gamma^{t}\right\}, D} \limsup _{T \rightarrow \infty} \frac{\sum_{t \leq T} O P T\left(\mathbf{v}^{t}, \gamma^{t}\right)}{\sum_{t \leq T} S W\left(\pi\left(\mathbf{b}^{t}, \gamma^{t}\right), \mathbf{v}^{t}, \gamma^{t}\right)} .
$$

As in the full information setting, there is a relationship between regret minimization under uncertainty and coarse correlated equilibria with uncertainty. Note however, that the speed of learning now depends on

\footnotetext{
${ }^{3}$ In fact, we can also think of the set of players as changing on each round: if player $i$ is assigned type 0 on a given round, this can be interpreted as player $i$ not being present in that round.

${ }^{4}$ For instance, one could assume that valuations are bounded and multiples of some arbitrarily small increment.
} 
the time needed for the empirical distribution of iid samples $\left(\mathbf{v}^{t}, \gamma^{t}\right)$ to resemble the original distribution and for learning algorithms to guarantee low regret with high probability. We discuss this in more detail in Section 5.2

\section{Semi-Smooth Games and the Price of Anarchy with Uncertainty}

Our main result is a bound on the price of anarchy for the Generalized Second Price auction with uncertainty. Recall that our model captures two types of uncertainty: uncertainty for player types and uncertainty about quality factors. Further, our result holds even in the presence of information asymmetry in the form of personalized signals available to the players 5 For simplicity of presentation, we focus on the setting where there are no signals and player valuations and quality factors are drawn from a known joint distribution $(\mathbf{F}, \mathbf{G})$.

Theorem 3.1 The price of anarchy of the Generalized Second Price auction with uncertainty is at most 2.927. That is, for any fixed click-through-rates $\alpha_{1}, \ldots, \alpha_{n}$, any joint distribution $(\mathbf{F}, \mathbf{G})$ over valuation profiles and quality factors, and any Bayes-Nash equilibrium $\mathbf{b}$,

$$
\mathbb{E}_{\mathbf{v}, \gamma, \mathbf{b}}[S W(\pi(\mathbf{b}, \gamma), \mathbf{v}, \gamma)] \geq \frac{1}{2.927} \mathbb{E}_{\mathbf{v}, \gamma}[O P T(\mathbf{v}, \gamma)]
$$

Semi-smooth games and the price of anarchy. Our proof is based on an extension of a proof technique introduced by Roughgarden [31], which he calls smoothness. We begin by reviewing this notion briefly in the context of a general game. Let $\mathbf{t}$ denote the (fixed) player types in a game, and $\mathbf{h}$ a pure strategy profile for the players, and let $U_{i}(\mathbf{t}, \mathbf{h})$ denote the utility of player $i$ with player types $\mathbf{t}$, and strategy profile $\mathbf{h}$. Let $s w(\mathbf{t}, \mathbf{h})$ denote the social welfare generated by strategy profile $\mathbf{h}$, and $s w^{*}(\mathbf{t})$ the maximum possible social welfare. Roughgarden defines $(\lambda, \mu)$-smooth games as games where for all pairs of pure strategy profiles $\mathbf{h}, \mathbf{h}^{\prime}$, and any (fixed) vector of types $\mathbf{t}$, we have

$$
\sum_{i} U_{i}\left(\mathbf{t}, h_{i}^{\prime}, \mathbf{h}_{-i}\right) \geq \lambda \cdot s w\left(\mathbf{t}, \mathbf{h}^{\prime}\right)-\mu \cdot s w(\mathbf{t}, \mathbf{h}) .
$$

Roughly speaking, smoothness captures the property that if strategy profile $\mathbf{h}^{\prime}$ results in a significantly larger social welfare than another strategy profile $\mathbf{h}$, then a large part of this gap in welfare is captured by the marginal increases in the utility of each individual player when unilaterally switching her strategy from $h_{i}$ to $h_{i}^{\prime}$.

It is not hard to see that GSP does not satisfy this definition for all pairs of strategy profiles $\mathbf{h}, \mathbf{h}^{\prime}$. However, we argue that GSP is smooth with respect to a particular (possibly randomized) strategy profile $\mathbf{h}^{\prime}$, as defined by Nadav and Roughgarden [27], that can be used by players unilaterally to improve the efficiency of GSP whenever its allocation resulting from a pure strategy profile $\mathbf{h}$ is highly inefficient. Note that unlike [27] we require improvement relative to the social optimum $s w^{*}(\mathbf{t})$ and not relative to $s w\left(\mathbf{t}, \mathbf{h}^{\prime}\right)$, i.e., we will not assume that $s w\left(\mathbf{t}, \mathbf{h}^{\prime}\right)$ is (close to) the maximum $s w^{*}(\mathbf{t})$. Further, we will show that there exists such a strategy profile $\mathbf{h}^{\prime}$ where the strategy $\mathbf{h}_{i}^{\prime}$ of a player depends only on the type of the player. We call games that satisfy this stronger requirement semi-smooth.

\footnotetext{
${ }^{5}$ In the presence of additional signals, we can assume that signal $s$ also encodes the valuation of the player, i.e., that player $i$ 's valuation for a click when she receives signal $s_{i}$ is $v_{i}\left(s_{i}\right)$, and in this case, signals and quality factors are drawn from a known joint distribution $\left(\mathbf{F}^{\prime}, \mathbf{G}\right)$. Our statement and proof carry over to this case with straightforward modifications.
} 
Definition 3.2 (semi-smooth games) We say that a game is $(\lambda, \mu)$-semi-smooth if for each player $i$ there exists some (possibly randomized) strategy $h_{i}^{\prime}(\cdot)$ (depending only on the type of the player) such that,

$$
\sum_{i} \mathbb{E}_{h_{i}^{\prime}\left(t_{i}\right)}\left[U_{i}\left(\mathbf{t}, h_{i}^{\prime}\left(t_{i}\right), \mathbf{h}_{-i}\right)\right] \geq \lambda \cdot s w^{*}(\mathbf{t})-\mu \cdot s w(\mathbf{t}, \mathbf{h}),
$$

for every pure strategy profile $\mathbf{h}$ and every (fixed) type vector $\mathbf{t}$. The expectation is taken over the random bits of $h_{i}^{\prime}\left(t_{i}\right)$.

Analogous to Roughgarden's [31] proof (see also Nadav and Roughgarden [27]), semi-smoothness also immediately implies a bound on the price of anarchy with uncertainty even when the types are arbitrarily correlated.

Lemma 3.3 If a game is $(\lambda, \mu)$-semi-smooth and its social welfare is at least the sum of the players' utilities, then the price of anarchy with uncertainty (and information asymmetries) is at most $(\mu+1) / \lambda$.

Proof. Consider a game in the Bayesian setting where player types are drawn from a joint probability distribution and let $\mathbf{h}$ be a Bayes-Nash equilibrium for this game. By the definition of the Bayes-Nash equilibrium, we have that $\mathbb{E}_{\mathbf{t}_{-i}, \mathbf{h}}\left[U_{i}(\mathbf{t}, \mathbf{h}) \mid t_{i}\right] \geq \mathbb{E}_{\mathbf{t}_{-i}, \mathbf{h}}\left[U_{i}\left(\mathbf{t}, h_{i}^{\prime}\left(t_{i}\right), \mathbf{h}_{-i}\right) \mid t_{i}\right]$ for every value the random variable $h_{i}^{\prime}\left(t_{i}\right)$ may take. Hence, $\mathbb{E}_{\mathbf{t}_{-i}, \mathbf{h}}\left[U_{i}(\mathbf{t}, \mathbf{h}) \mid t_{i}\right] \geq \mathbb{E}_{\mathbf{t}_{-i}, \mathbf{h}} \mathbb{E}_{h_{i}^{\prime}\left(t_{i}\right)}\left[U_{i}\left(\mathbf{t}, h_{i}^{\prime}\left(t_{i}\right), \mathbf{h}_{-i}\right) \mid t_{i}\right]$. Now taking expectation over $t_{i}$, we get $\mathbb{E}_{\mathbf{t}, \mathbf{h}}\left[U_{i}(\mathbf{t}, \mathbf{h})\right] \geq \mathbb{E}_{\mathbf{t}, \mathbf{h}} \mathbb{E}_{h_{i}^{\prime}\left(t_{i}\right)}\left[U_{i}\left(\mathbf{t}, h_{i}^{\prime}\left(t_{i}\right), \mathbf{h}_{-i}\right)\right]$. By summing over all players, and using the fact that the social welfare is at least the sum of the players' utilities, as well as the semismoothness property, we have

$$
\begin{aligned}
\mathbb{E}_{\mathbf{t}, \mathbf{h}}[s w(\mathbf{t}, \mathbf{h})] & \geq \mathbb{E}_{\mathbf{t}, \mathbf{h}}\left[\sum_{i} U_{i}(\mathbf{t}, \mathbf{h})\right] \\
& \geq \mathbb{E}_{\mathbf{t}, \mathbf{h}}\left[\sum_{i} \mathbb{E}_{h_{i}^{\prime}\left(t_{i}\right)}\left[U_{i}\left(\mathbf{t}, h_{i}^{\prime}\left(t_{i}\right), \mathbf{h}_{-i}\right)\right]\right] \\
& \geq \mathbb{E}_{\mathbf{t}, \mathbf{h}}\left[\lambda \cdot s w^{*}(\mathbf{t})-\mu \cdot s w(\mathbf{t}, \mathbf{h})\right] \\
& =\lambda \mathbb{E}_{\mathbf{t}}\left[s w^{*}(\mathbf{t})\right]-\mu \mathbb{E}_{\mathbf{t}, \mathbf{h}}[s w(\mathbf{t}, \mathbf{h})] .
\end{aligned}
$$

Note that the third inequality follows by applying the semi-smoothness property for every fixed type vector and every pure strategy profile that are simultaneous outcomes of the random vectors $\mathbf{t}$ and $\mathbf{h}$. The last inequality implies $\mathbb{E}_{\mathbf{t}}\left[s w^{*}(\mathbf{t})\right] \leq \frac{\mu+1}{\lambda} \mathbb{E}_{\mathbf{t}, \mathbf{h}}[s w(\mathbf{t}, \mathbf{h})]$, as claimed.

We remark that the proof holds without significant changes if we add information asymmetries in the game, i.e., if we assume that each player gets signals that reveal her type and refine her knowledge on the probability distributions of the types of the other players. The only change required is to define an extended type for each player, consisting of the player's original type composed with that player's signal, and use it in place of the original type.

A particular strength of Lemma 3.3 lies in the fact that it can provide bounds on the efficiency loss for Bayesian games even with correlated types (and, as we will see later in Section 5, under even more general equilibrium concepts) by examining substantially more restricted settings. In the context of GSP auction games, it allows us to focus on identifying a (possibly randomized) deviating bid strategy for each player (i.e., a bid $b_{i}^{\prime}$ for each player $i$ ) so that the semi-smoothness inequality holds for every fixed valuation vector $\mathbf{v}$ and pure bidding profile $\mathbf{b}$. By Lemma 3.3 , this then immediately implies a bound on the price of anarchy of GSP auction games with uncertainty and information asymmetries. 
Remark 3.4 It is maybe easier to interpret a deterministic special case of Lemma 3.3 where we require that Definition 3.2 holds for deterministic bids $h_{i}^{\prime}(\cdot)$. As a warmup in analyzing the GSP auction, we will show in Claim 3.6 that the bids $b_{i}^{\prime}=\frac{1}{2} v_{i}$ can serve to prove that the GSP auction is $(1 / 2,1)$-smooth, and hence has a price of anarchy of at most 4 . This bound of 4 on the price of anarchy shows that if the social welfare is less than a quarter of the maximum possible, there is a player $i$ who can deviate to $b_{i}^{\prime}=\frac{1}{2} v_{i}$, a natural shading of her bid, and improve her utility.

To improve the bound we will consider a deviating bid $b_{i}=\theta \cdot v_{i}$ for other constants $\theta \in(0,1)$. In fact, we will need to consider a random $\theta$ rather than a constant one. There are two ways to understand such a random bid: a direct conclusion is that sampling $\theta$ according to the prescribed distribution produces a good deviation in expectation, whenever welfare is low. But maybe a more natural interpretation is through the lenses of the probabilistic method, used in combinatorics to show that a certain object exists without finding it explicitly. If there exists a randomized deviation $b_{i}=\theta \cdot v_{i}$ that improves player $i$ 's utility, this implies that there exists a deterministic bid $\theta v_{i}$ that improves player $i$ 's utility.

The randomization on selecting the bid $h_{i}^{\prime}(\cdot)$ in Definition 3.2 gives us more flexibility to prove the semi-smoothness inequality with good parameters $\lambda$ and $\mu$ by defining appropriately the density function of $h_{i}^{\prime}$ 's.

Price of anarchy of GSP auctions. First note that, technically speaking, the GSP auction does not immediately fit into the framework of semi-smoothness: advertiser payoffs depend on random quality factors which may be correlated with the type profile. However, this notational technicality is easily addressed by expressing advertiser utilities in expectation over quality scores. That is, expressing utilities in the GSP auction in the notation of general games, we have $U_{i}(\mathbf{v}, \mathbf{b})=\mathbb{E}_{\gamma}\left[u_{i}(\mathbf{b}, \gamma) \mid \mathbf{v}\right]$. Since quality factors affect the social welfare as well, we have $s w^{*}(\mathbf{v})=\mathbb{E}_{\gamma}[O P T(\mathbf{v}, \gamma) \mid \mathbf{v}]$ and $s w(\mathbf{v}, \mathbf{b})=\mathbb{E}_{\gamma}[S W(\pi(\mathbf{b}, \gamma), \mathbf{v}, \gamma) \mid \mathbf{v}]$.

We are ready to prove that GSP auction games are semi-smooth. We start by presenting a slightly weaker version of Theorem 3.1, where we prove a bound of 3.164. Then we sketch the proof of the improved bound of 2.927, which is more technically involved. Details of the proof can be found in Appendix $\mathrm{A}$.

Lemma 3.5 The GSP auction game is $\left(1-\frac{1}{e}, 1\right)$-semi-smooth.

Proof. We begin by rewriting the definition of semi-smoothness in the notation of GSP auctions. The GSP auction game is $\left(1-\frac{1}{e}, 1\right)$-semi-smooth if and only if, for each valuation profile $\mathbf{v}$, there exists a (possibly randomized) bid profile $\mathbf{b}^{\prime}$ (with $b_{i}^{\prime}$ depending only on the valuation of player $i$ ) such that, for every bid profile $\mathbf{b}$,

$$
\sum_{i} \mathbb{E}_{\gamma, b_{i}^{\prime}}\left[u_{i}\left(b_{i}^{\prime}, \mathbf{b}_{-i}, \gamma\right) \mid \mathbf{v}\right] \geq\left(1-\frac{1}{e}\right) \mathbb{E}_{\gamma}[O P T(\mathbf{v}, \gamma) \mid \mathbf{v}]-\mathbb{E}_{\gamma}[S W(\pi(\mathbf{b}, \gamma), \mathbf{v}, \gamma) \mid \mathbf{v}]
$$

We will actually establish the stronger property that this inequality holds for all $\gamma$, and not only in expectation.

$$
\sum_{i} \mathbb{E}_{b_{i}^{\prime}}\left[u_{i}\left(b_{i}^{\prime}, \mathbf{b}_{-i}, \gamma\right)\right] \geq\left(1-\frac{1}{e}\right) O P T(\mathbf{v}, \gamma)-S W(\pi(\mathbf{b}, \gamma), \mathbf{v}, \gamma)
$$

The desired inequality (1) will then follow by taking (2) in expectation over the choice of $\gamma$ (whose distribution may depend on the valuation profile $\mathbf{v}$ ).

Before establishing inequality (2), we will prove the even weaker statement that the GSP auction game is $(1 / 2,1)$-semi-smooth (which implies a bound of 4 on the price of anarchy with uncertainty). 
Claim 3.6 The GSP auction game is $(1 / 2,1)$-semi-smooth.

Proof. Choose a vector $\mathbf{v}$ of fixed valuations, a pure bidding profile $\mathbf{b}$, and quality factors $\gamma$. Consider a (deterministic) deviating bid $b_{i}^{\prime}=v_{i} / 2$ for each player $i$. We distinguish between two cases (recalling that $\nu(i)$ is the slot assigned to player $i$ in the efficient allocation given $\mathbf{v}$ and $\gamma$ ):

- If by bidding $b_{i}^{\prime}$ player $i$ gets slot $\nu(i)$ or better, then $u_{i}\left(b_{i}^{\prime}, \mathbf{b}_{-i}, \gamma\right) \geq \alpha_{\nu(i)} \gamma_{i} v_{i} / 2$, as the payment $p_{i}$ cannot exceed her effective bid.

- If by bidding $b_{i}^{\prime}$ player $i$ gets a slot lower than $\nu(i)$, then the effective value of the player $\pi(\nu(i))$ in slot $\nu(i)$ is at least $\gamma_{i} v_{i} / 2$, as we assume no overbidding.

We conclude that, in either case,

$$
u_{i}\left(b_{i}^{\prime}, \mathbf{b}_{-i}, \gamma\right) \geq \alpha_{\nu(i)} \gamma_{i} v_{i} / 2-\alpha_{\nu(i)} \gamma_{\pi(\nu(i))} v_{\pi(\nu(i))} .
$$

Summing over all players, and noticing that $\sum_{i} \alpha_{i} \gamma_{\pi(i)} v_{\pi(i)}=S W(\pi(\mathbf{b}, \gamma), \mathbf{v}, \gamma)$, while $\sum_{i} \alpha_{\nu(i)} \gamma_{i} v_{i}=$ $\operatorname{OPT}(\mathbf{v}, \gamma)$, we arrive at the claimed bound that the GSP auction game is $(1 / 2,1)$-semi-smooth:

$$
\sum_{i} u_{i}\left(b_{i}^{\prime}, \mathbf{b}_{-i}, \gamma\right) \geq \frac{1}{2} O P T(\mathbf{v}, \gamma)-S W(\pi(\mathbf{b}, \gamma), \mathbf{v}, \gamma)
$$

Notice that the proof uses a single Nash inequality: that no player $i$ would be better off changing her bid to $b_{i}^{\prime}=v_{i} / 2$, bidding half her valuation, a natural shading of her valuation. As we will see in Section 5 , the bound will also apply to learning outcomes under the same assumption of not regretting this single alternative.

Now we return to proving the $\left(1-\frac{1}{e}, 1\right)$ semi-smoothness. To do this, consider a randomized bid $\mathbf{b}^{\prime}$, rather than the deterministic bid of $b_{i}^{\prime}=v_{i} / 2$ considered above, that offers a more sophisticated bid-shading strategy. We consider a random strategy where player $i$ shades her bid randomly to a value in the interval $\left[0, v_{i}\left(1-\frac{1}{e}\right)\right]$, where bid $b_{i}^{\prime}$ is a random variable with density $f(y)=\frac{1}{v_{i}-y}$ for $y \in\left[0, v_{i}\left(1-\frac{1}{e}\right)\right]$ and $f(y)=0$ otherwise. We will show that

$$
\mathbb{E}_{b_{i}^{\prime}}\left[u_{i}\left(b_{i}^{\prime}, \mathbf{b}_{-i}, \gamma\right)\right] \geq\left(1-\frac{1}{e}\right) \alpha_{\nu(i)} \gamma_{i} v_{i}-\alpha_{\nu(i)} \gamma_{\pi(\nu(i))} b_{\pi(\nu(i))} .
$$

Like in the proof of Claim 3.6, by summing expression (3) for all $i$ and using the fact that $b_{\pi(i)} \leq v_{\pi(i)}$ by the non-overbidding assumption, we obtain that the game is $\left(1-\frac{1}{e}, 1\right)$-semi-smooth.

It remains to derive equation (3). We have that

$$
\begin{aligned}
\mathbb{E}_{b_{i}^{\prime}}\left[u_{i}\left(b_{i}^{\prime}, \mathbf{b}_{-i}, \gamma\right)\right] & \geq \mathbb{E}_{b_{i}^{\prime}}\left[\alpha_{\nu(i)} \gamma_{i}\left(v_{i}-b_{i}^{\prime}\right) \mathbb{1}\left\{\gamma_{i} b_{i}^{\prime} \geq \gamma_{\pi(\nu(i))} b_{\pi(\nu(i))}\right\}\right] \\
& =\int_{0}^{v_{i}\left(1-\frac{1}{e}\right)} \alpha_{\nu(i)} \gamma_{i}\left(v_{i}-y\right) \mathbb{1}\left\{\gamma_{i} y \geq \gamma_{\pi(\nu(i))} b_{\pi(\nu(i))}\right\} \frac{1}{v_{i}-y} d y \\
& =\alpha_{\nu(i)} \gamma_{i}\left[v_{i}\left(1-\frac{1}{e}\right)-\frac{\gamma_{\pi(\nu(i))}}{\gamma_{i}} b_{\pi(\nu(i))}\right]^{+} \\
& \geq\left(1-\frac{1}{e}\right) \alpha_{\nu(i)} \gamma_{i} v_{i}-\alpha_{\nu(i)} \gamma_{\pi(\nu(i))} b_{\pi(\nu(i))}
\end{aligned}
$$

which implies (3), completing the proof of Lemma 3.5.

Combining Lemmas 3.3 and 3.5, we get the claimed bound on the price of anarchy. 
Theorem 3.7 The price of anarchy of the Generalized Second Price auction with uncertainty (and with information asymmetries) is at most $2(1-1 / e)^{-1} \approx 3.164$.

To prove Theorem 3.1 we will need to extend semi-smoothness specially tailored to the GSP auction game. In addition to working more carefully on optimizing constants, we want to highlight two ideas: First, note that the payment of the player in slot $i$ is $\alpha_{i} \gamma_{\pi(i+1)} b_{\pi(i+1)}$, and this payment also contributes to the social welfare. Using that $\alpha$ is monotone decreasing, we can add a term $\alpha_{i} \gamma_{\pi(i)} b_{\pi(i)}$ to social welfare (in addition to the advertisers' utility) for all slots except the top one. Second, the player in the top slot can obtain a stronger bound on her utility by considering the deviation $b_{1}^{\prime}=v_{1}$. (For all other players bidding too close to $v_{i}$ endangers getting a higher slot at too high a price, but the top player does not face this danger.) We will use these ideas in Section 5 to improve our bound on the learning outcomes for full information games. The details of the more complicated improved bound for the Bayesian case are found in Appendix A

Discretization of the bidding space. Analogous results also hold when the possible bid space is discretized (i.e., players need to bid in integer number of pennies). With a finely enough discretized bid space, the players could approximately follow the bidding strategies used in the above proofs, as well as in the proofs in Appendix A The Nash property then implies that the same bound holds at the equilibria with a small loss due to the discretization. Recall that this case is both of practical relevance, and using a result of Athey [2] and Reny [30], in the discrete case if player types and quality factors are drawn independently, the existence of pure strategy equilibria that are monotone in the types is also guaranteed.

In order to illustrate this point, we show how to adapt Lemma 3.5 and Theorem 3.7 to the case where possible bids are discrete. Assume bids $b_{i}$ must be in the finite set $T_{\epsilon, K}=\{0, \epsilon, 2 \epsilon, \ldots, K \epsilon\}$ for some large integer $K$. We also need to assume that $\epsilon$ is small compared to the valuations. We will assume that $\epsilon<\frac{1}{e}$ and all types in the support of the distribution are $v_{i} \geq 1$. We show that:

Lemma 3.8 The GSP auction game with discretized bid is $\left(\left(1-\frac{1}{e}\right)(1-e \epsilon), 1-e \epsilon\right)$-semi-smooth assuming $\epsilon<\frac{1}{e}$ and $v_{i} \geq 1$ for all $i$. That is, there is a deviation $b_{i}^{\prime}$ from the discrete space $T_{\epsilon, K}$ that satisfies the semi-smoothness inequality.

Proof. The proof follows from a small modification of Lemma 3.5. There we considered the deviation where a player with valuation $v_{i}$ samples a bid $b_{i}^{\prime}$ from the distribution with density $f(y)=\frac{1}{v_{i}-y}$ for $y \in\left[0,\left(1-\frac{1}{e}\right) v_{i}\right]$. In this setting, bids must lie in $T_{\epsilon, K}$, so we use a rounded version instead: $\hat{b}_{i}^{\prime}=\epsilon \cdot\left\lceil\frac{y}{\epsilon}\right\rceil$. This change increases the probability that $\gamma_{i} \hat{b}_{i}^{\prime} \geq \gamma_{\pi(\nu(i))} b_{\pi(\nu(i))}$, but decreases the expression $v_{i}-b_{i}^{\prime}$ inside the integral. This decrease, however, is bounded since it holds that

$$
\min _{y} \frac{v_{i}-\epsilon \cdot\left\lceil\frac{y}{\epsilon}\right\rceil}{v_{i}-y} \geq\left(1-\frac{\epsilon e}{v_{i}}\right) \geq 1-\epsilon e .
$$

Using the same calculation as in the proof of Lemma 3.5, we get that

$$
\mathbb{E}_{\hat{b}_{i}^{\prime}}\left[u_{i}\left(\hat{b}_{i}^{\prime}, \mathbf{b}_{-i}, \gamma\right)\right] \geq(1-\epsilon e)\left(1-\frac{1}{e}\right) \alpha_{\nu(i)} \gamma_{i} v_{i}-(1-\epsilon e) \alpha_{\nu(i)} \gamma_{\pi(\nu(i))} b_{\pi(\nu(i))} .
$$

Using this Lemma 3.8 we immediately get the following theorem. 
Theorem 3.9 The price of anarchy of the Generalized Second Price auction with uncertainty and discretized bids is at most $\left(1+\frac{1}{1-\epsilon e}\right) \cdot\left(1-\frac{1}{e}\right)^{-1}=3.16+O(\epsilon)$, assuming that bids lie on $T_{\epsilon, K}=\{0, \epsilon, 2 \epsilon, \ldots, K \epsilon\}$, for a large integer $K$ and $\epsilon<1 / e$, and that, for each player $i, v_{i} \geq 1$.

\section{Pure Nash Equilibria in the Full Information Setting}

In this section we turn our attention to the full information setting, where the quality factors $\gamma$ are fixed and common knowledge. Without loss of generality we can assume that $\gamma_{1} v_{1} \geq \gamma_{2} v_{2} \geq \ldots \geq \gamma_{n} v_{n}$. In this setting the strategy of a player is a single bid $b_{i} \in\left[0, v_{i}\right]$, again assuming that players do not overbid. Our main result in this setting is the following:

Theorem 4.1 The (pure) price of anarchy of the Generalized Second Price auction in the full information setting is at most 1.282. In other words, for any fixed click-through-rates $\alpha$, valuation profile $\mathbf{v}$, and quality factors $\gamma$, if $\mathbf{b}$ is a bid profile in pure Nash equilibrium, then $S W(\pi(\mathbf{b}), \mathbf{v}) \geq \frac{1}{1.282} \cdot O P T(\mathbf{v}) \approx 0.78$. $O P T(\mathbf{v})$.

The bound above is very close to being tight, since we can exhibit an example with 3 players and 3 slots for which there is an equilibrium where the gap between the optimal social welfare and the social welfare in equilibrium is 1.259. Also, we can show the following slightly stronger bound for a small number of players and slots. Notice however that the bound in Theorem 4.1 holds regardless of the number of slots.

Theorem 4.2 For 2 players and 2 slots, the price of anarchy is exactly 1.25. For 3 players and 3 slots, the price of anarchy is exactly 1.259. By exactly we mean that there is a particular GSP auction game with an equilibrium matching this bound.

Proof. Here we give an example with two slots that yields price of anarchy 1.25. In Appendix B, we show that this is worst possible, and show the bound for 3 slots.

For two slots, consider an example with two players with valuations 1 and $1 / 2$ respectively, quality factors $\gamma_{1}=\gamma_{2}=1$, and two slots with $\alpha_{1}=1$ and $\alpha_{2}=1 / 2$. The bids $b_{1}=0$ and $b_{2}=1 / 2$ are at equilibrium, resulting in a social welfare of 1 , while the optimal social welfare is 1.25 .

The full proof of Theorem 4.1 can be found in Appendix B Here instead, we present the proof of a weaker bound that highlights the intuition underlying our result that GSP equilibria have good social welfare properties.

Theorem 4.3 The (pure) price of anarchy of the Generalized Second Price auction in the full information setting is at most 2 .

The proof is based on the concept of weakly feasible allocations. Recall that each bid profile $\mathbf{b}$ defines an allocation $\pi$ that is a mapping from slots to players $\pi:[n] \rightarrow[n]$.

Definition 4.4 (weakly feasible allocations) We say that an allocation $\pi$ is weakly feasible if the following holds for each pair $i, j$ of slots:

$$
\frac{\alpha_{j}}{\alpha_{i}}+\frac{\gamma_{\pi(i)} v_{\pi(i)}}{\gamma_{\pi(j)} v_{\pi(j)}} \geq 1
$$


We also use the term weak feasibility condition to refer to inequality (4).

The concept of weakly feasible allocations is a relaxation of the concept of Nash equilibrium. We adopt this terminology to denote it is a weakening of the feasibility conditions for Nash equilibrium. This concept encapsulates the fact that an allocation in equilibrium cannot be too far from the optimal. The optimal allocation is such that $\pi(i)=i$, since both $\left\{\alpha_{i}\right\}$ and $\left\{\gamma_{i} v_{i}\right\}$ are sorted. If an allocation is not optimal, then two slots $i<j$ have advertisers assigned to them such that $\pi(i)>\pi(j)$, i.e., they are assigned in the wrong order. Equation (4) implies that at least one of the two ratios is at least $1 / 2$, and hence whenever advertisers are assigned in the non-optimal order, then either (i) the two advertisers have similar effective values for a click, or (ii) the click-through-rates of the two slots are not very different; in either case their relative order does not affect the social welfare very much.

Lemma 4.5 If $\mathbf{b}$ is a Nash equilibrium of the GSP auction game, then the induced allocation $\pi$ satisfies the weak feasibility condition.

Proof. If $j \leq i$ the inequality is obviously true. Otherwise consider the player $\pi(j)$ in slot $j$. Since $\mathbf{b}$ is a Nash equilibrium, the player in slot $j$ is happy with her outcome and does not want to increase her bid to take slot $i$, so: $\alpha_{j}\left(\gamma_{\pi(j)} v_{\pi(j)}-\gamma_{\pi(j+1)} b_{\pi(j+1)}\right) \geq \alpha_{i}\left(\gamma_{\pi(j)} v_{\pi(j)}-\gamma_{\pi(i)} b_{\pi(i)}\right)$ since $b_{\pi(j+1)} \geq 0$ and $b_{\pi(i)} \leq v_{\pi(i)}$ then: $\alpha_{j} \gamma_{\pi(j)} v_{\pi(j)} \geq \alpha_{i}\left(\gamma_{\pi(j)} v_{\pi(j)}-\gamma_{\pi(i)} v_{\pi(i)}\right)$.

Given Lemma 4.5, the proof of Theorem 4.3 follows almost directly:

Proof of Theorem 4.3. Taking $j=\sigma(i)$ in the definition of weakly feasible allocations, we get that: $\alpha_{\sigma(i)} \gamma_{i} v_{i}+\alpha_{i} \gamma_{\pi(i)} v_{\pi(i)} \geq \alpha_{i} \gamma_{i} v_{i}$. Now, summing this for each player $i$, we get

$$
2 \cdot S W(\pi(\mathbf{b}), \mathbf{v})=\sum_{i} \alpha_{\sigma(i)} \gamma_{i} v_{i}+\sum_{i} \alpha_{i} \gamma_{\pi(i)} v_{\pi(i)} \geq \sum_{i} \alpha_{i} \gamma_{i} v_{i}=O P T(\mathbf{v}) .
$$

To prove Theorem 4.1 we proceed by induction on the number of slots. Given an allocation $\pi$, consider the directed graph $G(\pi)$ that has one node for each slot, and a directed edge for each advertiser $i$ that connects the node corresponding to slot $i$ to the node corresponding to slot $\pi^{-1}(i)$. When the allocation is optimal, this graph consists of self-loops. In general, $G(\pi)$ consists of a set of disjoint cycles, however, without loss of generality, we can assume $G(\pi)$ is a single cycle. We obtain the improved bound by considering four nodes in the neighborhood of node 1 in this cycle, and separately considering cases depending on the order of the effective values of the corresponding players. The details of the proof can be found in Appendix B

\section{Quality of Learning Outcomes in GSP}

In this section, we bound the average quality of outcomes in a repeated play of a GSP auction game where players employ strategies that guarantee no external regret. In both the full information setting and the setting with uncertainty, we can reduce the problem over declaration sequences to a problem over distributions. This will allow us to adapt our earlier bounds on the price of anarchy from Sections 3 and 4 to bound the price of total anarchy. As in previous sections, we show simple and intuitive bounds in this section, and defer improved and more complex bounds to the appendix. 


\subsection{Learning in the full information setting}

We will first focus upon the full information setting of the GSP auction. Recall that, in this model, the valuation profile $\mathbf{v}$ and quality factors $\gamma$ are fixed and common knowledge. As in the previous section, we will assume that $\gamma_{1} v_{1} \geq \gamma_{2} v_{2} \geq \ldots \geq \gamma_{n} v_{n}$.

We will begin by proving a relationship between the price of total anarchy and the set of coarse correlated equilibria for the GSP auction in the full information model. Given a valuation profile $\mathbf{v}$, a distribution $\mathbf{D}$ over bid profiles is called a coarse correlated equilibrium if

$$
\mathbb{E}_{\mathbf{b} \sim \mathbf{D}}\left[u_{i}(\mathbf{b})\right] \geq \mathbb{E}_{\mathbf{b} \sim \mathbf{D}}\left[u_{i}\left(b_{i}^{\prime}, \mathbf{b}_{-i}\right)\right], \forall i, b_{i}^{\prime} .
$$

As we shall show, the price of total anarchy can be bounded by considering the social welfare generated at any coarse correlated equilibrium.

Lemma 5.1 The price of total anarchy in the full information setting is at most

$$
\sup _{\mathbf{v}, \mathbf{D} \in C C E} \frac{O P T(\mathbf{v})}{\mathbb{E}_{\mathbf{b} \sim \mathbf{D}}[S W(\pi(\mathbf{b}), \mathbf{v})]}
$$

where $C C E$ is the set of coarse correlated equilibria.

Proof. Consider a declaration sequence $D=\left(\mathbf{b}^{1}, \ldots, \mathbf{b}^{t}, \ldots\right)$ in the full information case. For each $T$ let $\mathbf{D}^{T}$ be the distribution over bid profiles where each $\mathbf{b}^{t}$ for $t \leq T$ is drawn with probability $\frac{1}{T}$. Proving that the price of total anarchy is bounded by $\eta$ is equivalent to showing that:

$$
\liminf _{T} \mathbb{E}_{\mathbf{b} \sim \mathbf{D}^{T}}[S W(\pi(\mathbf{b}), \mathbf{v})] \geq \frac{1}{\eta} O P T(\mathbf{v}) .
$$

Since the set of all possible bid profiles is compact, one needs to prove that for all distributions $\mathbf{D}$ such that there is a subsequence of $\left\{\mathbf{D}^{T}\right\}_{T}$ converging in distribution to $\mathbf{D}$ we have:

$$
\mathbb{E}_{\mathbf{b} \sim \mathbf{D}}[S W(\pi(\mathbf{b}), \mathbf{v})] \geq \frac{1}{\eta} O P T(\mathbf{v}) .
$$

It is therefore sufficient to show that such a $\mathbf{D}$ is a coarse correlated equilibrium. We note that the fact that the declaration sequence $D$ minimizes external regret implies that, for each distribution $\mathbf{D}$ which can be written as the limit of a subsequence of $\left\{\mathbf{D}^{T}\right\}_{T}$, it holds that:

$$
\mathbb{E}_{\mathbf{b} \sim \mathbf{D}}\left[u_{i}(\mathbf{b})\right] \geq \mathbb{E}_{\mathbf{b} \sim \mathbf{D}}\left[u_{i}\left(b_{i}^{\prime}, \mathbf{b}_{-i}\right)\right], \forall i, b_{i}^{\prime}
$$

as required.

Using this connection to coarse correlated equilibria, we are able to obtain a bound of 2.310 on the price of total anarchy of the GSP auction.

Theorem 5.2 The price of total anarchy of the Generalized Second Price auction in the full information setting is at most 2.310 .

A full proof of Theorem 5.2 appears in Appendix C. We now present a simpler proof of the following weaker bound, which captures some of the intuition behind the proof of Theorem 5.2 
Theorem 5.3 The price of total anarchy of the Generalized Second Price auction in the full information setting is at most 3.

Proof. The proof can be thought of as an improved version of the price of anarchy bound based on the fact that the GSP auction game is $(1 / 2,1)$-semi-smooth. We consider a distribution $\mathbf{D}$ which corresponds to a coarse correlated equilibrium. All expectations in the following are taken with respect to $\mathbf{b} \sim \mathbf{D}$. Recall the outline of the bound of 4 on the price of anarchy based on the fact that the GSP auction game is $(1 / 2,1)$-semi-smooth. We considered a possible deviation for player $i$ with valuation $v_{i}$ to bid $b_{i}^{\prime}=v_{i} / 2$, and concluded the bound $u_{i}\left(b_{i}^{\prime}, \mathbf{b}_{-i}\right) \geq \alpha_{i} \gamma_{i} v_{i} / 2-\alpha_{i} \gamma_{\pi(i)} b_{\pi(i)}$ in the proof of Lemma 3.5. We use the no-regret inequality directly, to get that

$$
\mathbb{E}\left[u_{i}(\mathbf{b})\right] \geq \alpha_{i} \gamma_{i} v_{i} / 2-\mathbb{E}\left[\alpha_{i} \gamma_{\pi(i)} b_{\pi(i)}\right] .
$$

Using that $b_{\pi(i)} \leq v_{\pi(i)}$, and summing over all players we get a bound of 4 on the price of total anarchy as was done in Lemma 3.3

Here we improve this bound by adding two new ideas. First, note that for all slots except the top one $\alpha_{i} \gamma_{\pi(i)} b_{\pi(i)}$ is a lower bound to the payment of the player in slot $i-1$. The social welfare is the sum of player utilities and the payments. The inequality states that in expectation the utility of player $i$ plus the payment of the player in slot $i-1$ is at least $\alpha_{i} \gamma_{i} v_{i} / 2$, i.e., half of the social welfare contributed by player $i$ in the efficient solution. To turn this into a bound on social welfare, we need to handle player 1 differently, as $\alpha_{1} \gamma_{\pi(1)} b_{\pi(1)}$ does not correspond to any payment.

The second observation is that for player 1 we can obtain a stronger bound on her utility by considering the deviation $b_{1}^{\prime}=v_{1}$. For other players such a high bid would endanger them to get a slot much higher than their slot in the optimum at a very high price. But player 1 already gets the best slot in the efficient solution. Deviating to $b_{1}^{\prime}=v_{1}$ will give the player the top slot, and hence utility $\alpha_{1} \gamma_{1} v_{1}-\alpha_{1} \gamma_{\pi(1)} b_{\pi(1)}$. Now using the no-regret property we get

$$
\mathbb{E}\left[u_{1}(\mathbf{b})\right] \geq \alpha_{1} \gamma_{1} v_{1}-\mathbb{E}\left[\alpha_{1} \gamma_{\pi(1)} b_{\pi(1)}\right]
$$

By summing over all players and writing the social welfare as the sum of utilities plus the total payments, we get:

$$
\begin{aligned}
& \mathbb{E}[S W(\pi(\mathbf{b}), \mathbf{v})]=\mathbb{E}\left[\sum_{i} u_{i}(\mathbf{b})\right]+\mathbb{E}\left[\sum_{i} \alpha_{i} \gamma_{\pi(i+1)} b_{\pi(i+1)}\right] \\
& \quad \geq \frac{1}{2} \mathbb{E}\left[u_{1}(\mathbf{b})\right]+\sum_{i \geq 2} \mathbb{E}\left[u_{i}(\mathbf{b})\right]+\mathbb{E}\left[\sum_{i \geq 2} \alpha_{i} \gamma_{\pi(i)} b_{\pi(i)}\right] \\
& \quad \geq \frac{\alpha_{1} \gamma_{1} v_{1}}{2}-\frac{\mathbb{E}\left[\alpha_{1} \gamma_{\pi(1)} b_{\pi(1)}\right]}{2}+\sum_{i \geq 2} \frac{\alpha_{i} \gamma_{i} v_{i}}{2}-\sum_{i \geq 2} \mathbb{E}\left[\alpha_{i} \gamma_{\pi(i)} b_{\pi(i)}\right]+\sum_{i \geq 2} \mathbb{E}\left[\alpha_{i} \gamma_{\pi(i)} b_{\pi(i)}\right] \\
& \quad=\frac{1}{2} O P T(\mathbf{v})-\frac{1}{2} \mathbb{E}\left[\alpha_{1} \gamma_{\pi(1)} b_{\pi(1)}\right] .
\end{aligned}
$$

Since $\mathbb{E}[S W(\pi(\mathbf{b}), \mathbf{v})] \geq \mathbb{E}\left[\alpha_{1} \gamma_{\pi(1)} b_{\pi(1)}\right]$, we obtain that $\mathbb{E}[S W(\pi(\mathbf{b}), \mathbf{v})] \geq \frac{1}{3} O P T(\mathbf{v})$. 


\subsection{Learning with uncertainty}

Let us now turn to the model of learning outcomes with uncertainty. As in the full information model, we can define a Bayesian version of the coarse correlated equilibrium. A Bayesian coarse correlated equilibrium is a joint distribution $(\mathbf{v}, \gamma, \mathbf{b})$ whose $(\mathbf{v}, \gamma)$-marginals are $(\mathbf{F}, \mathbf{G})$ and satisfies the following property:

$$
\mathbb{E}_{(\mathbf{v}, \gamma, \mathbf{b})}\left[u_{i}(\mathbf{b}, \gamma) \mid v_{i}\right] \geq \mathbb{E}_{(\mathbf{v}, \gamma, \mathbf{b})}\left[u_{i}\left(b_{i}^{\prime}, \mathbf{b}_{-i}, \gamma\right) \mid v_{i}\right], \forall i, v_{i}, b_{i}^{\prime} .
$$

Similarly to Lemma 5.1, we can show that the price of total anarchy with uncertainty can be bounded by considering the social welfare generated at any Bayesian coarse correlated equilibrium.

Lemma 5.4 Assuming that the distribution over types has finite support, the price of total anarchy with uncertainty is at most

$$
\sup _{\mathbf{F}, \mathbf{G}, \mathbf{D}(\cdot) \in C C E} \frac{\mathbb{E}_{\mathbf{v}, \gamma} O P T(\mathbf{v}, \gamma)}{\mathbb{E}_{\mathbf{v}, \gamma, \mathbf{b} \sim \mathbf{D}(\mathbf{v})}[S W(\pi(\mathbf{b}), \mathbf{v}, \gamma)]}
$$

where $C C E$ is the set of Bayesian coarse correlated equilibria.

Proof Sketch. The proof follows the same lines as the proof of Lemma 5.1 For each $t \geq 1,\left(\mathbf{v}^{t}, \gamma^{t}, \mathbf{b}^{t}\right)$ is the tuple of profiles corresponding to round $t$. Since the distribution over types has finite support, there is almost surely some $T_{0}$ such that, for each type profile $\tilde{\mathbf{v}}$ in the support of $\mathbf{F}$, there is $t \leq T_{0}$ such that $\mathbf{v}^{t}=\tilde{\mathbf{v}}$. For each $T \geq T_{0}$, let $\mathbf{D}^{T}$ be the joint distribution on $(\mathbf{v}, \gamma, \mathbf{b})$ that samples $t$ uniformly from $\{1,2, \ldots, T\}$ and outputs $\left(\mathbf{v}^{t}, \gamma^{t}, \mathbf{b}^{t}\right)$. This defines a sequence of distributions $\left\{\mathbf{D}^{T}\right\}_{T \geq T_{0}}$. Now, it is enough to observe that each convergent subsequence converges to a Bayesian coarse correlated equilibrium. Therefore the price of total anarchy is bounded by the price of anarchy over Bayesian coarse correlated equilibria.

Remark 5.5 Since our theorems hold in the limit as $T$ goes to infinity, they do not depend on the speed of learning - which we can define as the speed in which subsequences of $\left\{\mathbf{D}^{T}\right\}_{T}$ converge to a Bayesian coarse correlated equilibrium in the proof above. The rate of convergence depends on the specific learning methods being used by the players. However, the reader might notice that, regardless of the learning methods used, the speed of learning will depend on the time required for the empirical distribution of $\mathbf{v}, \gamma$ to resemble the real distribution. The speed in which this happens is controlled, for example, by the Central Limit Theorem. Also, if players observe only realized payoffs each round (rather than expectations), one would expect low click-through rates to increase the amount of time needed for learning, since more rounds will be required to accurately estimate expected outcomes. See Auer et al. [4] for a more detailed discussion on the speed of convergence of no-regret algorithms with limited feedback.

The arguments in the proof of Lemma 3.3 can be used with essentially no change to show that $(\lambda, \mu)$ semi-smoothness implies a bound of $(\mu+1) / \lambda$ to the price of total anarchy with uncertainty. From this, we know that:

Theorem 5.6 The price of total anarchy of the Generalized Second Price auction with uncertainty is bounded by 3.164 .

In Appendix A we present an improved result for the Bayesian price of anarchy that also extends to the following improved bound for learning outcomes.

Theorem 5.7 The price of total anarchy of the Generalized Second Price auction with uncertainty is bounded by 2.927 . 


\section{References}

[1] S. Arora, E. Hazan, and S. Kale. The multiplicative weights update method: a meta-algorithm and some applications. Theory of Computing Journal, 8(6): 121-164, 2012.

[2] S. Athey. Single crossing properties and the existence of pure strategy equlibria in games of incomplete information. Econometrica, 69(4): 861-890, 2001.

[3] S. Athey and D. Nekipelov. A structural model of sponsored search advertising auctions. Working paper. Preliminary version in 6th Workshop on Ad Auctions (AdAuctions '10), 2010.

[4] P. Auer, N. Cesa-Bianchi, Y. Freund, and R. E. Schapire. Gambling in a rigged casino: the adversarial multi-armed bandit problem. In Proceedings of the 36th Annual Symposium on Foundations of Computer Science (FOCS '95), pp. 322-331, 1995.

[5] K. Bhawalkar and T. Roughgarden. Welfare guarantees for combinatorial auctions with item bidding. In Proceedings of the 22nd Annual ACM-SIAM Symposium on Discrete Algorithms (SODA '11), pp. 700-709, 2011.

[6] A. Blum, M. Hajiaghayi, K. Ligett, and A. Roth. Regret minimization and the price of total anarchy. In Proceedings of the 40th Annual ACM Symposium on Theory of Computing (STOC '08), pp. 373-382, 2008.

[7] A. Blum and Y. Mansour. Learning, regret minimization, and equilibria. In N. Nisan, T. Roughgarden, É. Tardos, and V. V. Vazirani, editors, Algorithmic Game Theory, pp. 79-101. Cambridge University Press, 2007.

[8] I. Caragiannis, C. Kaklamanis, P. Kanellopoulos, and M. Kyropoulou. On the efficiency of equilibria in generalized second price auctions. In Proceedings of the 12th ACM Conference on Electronic Commerce (EC'11), pp. 81-90, 2011.

[9] G. Christodoulou, A. Kovács, and M. Schapira. Bayesian combinatorial auctions. In Proceedings of the 35th International Colloquium on Automata, Languages and Programming (ICALP '08), Part I, LNCS 5125, Springer, pp. 820-832, 2008.

[10] J. Cremer and R. P. McLean. Full extraction of the surplus in Bayesian and dominant strategy auctions. Econometrica, 56(6): 1247-1257, 1988.

[11] B. Edelman, M. Ostrovsky, and M. Schwarz. Internet advertising and the generalized second-price auction: Selling billions of dollars worth of keywords. American Economic Review, 97(1): 242-259, 2007.

[12] B. Edelman and M. Schwarz. Optimal auction design and equilibrium selection in sponsored search auctions. American Economic Review, 100(2): 597-602, 2010.

[13] eMarketer. Online ad spend continues double-digit growth. http://www.emarketer.com/Article.aspx?R=1008087

[14] D. P. Foster and R. V. Vohra. Calibrated learning and correlated equilibrium. Games and Economic Behavior, 21: 40-55, 1997. 
[15] D. Fudenberg and D. Levine. Conditional universal consistency. Games and Economic Behavior, 29: 104-130, 1999.

[16] R. D. Gomes and K. S. Sweeney. Bayes-Nash equilibria of the generalized second price auction. In Games and Economic Behavior, to appear.

[17] J. Hannan. Approximation to Bayes risk in repeated play. In M. Dresher, A. Tucker, and P. Wolfe, editors, Contributions to the Theory of Games, Volume 3. Princeton University Press, 1957.

[18] S. Hart and A. M. Colell. A simple adaptive procedure leading to correlated equilibrium. Econometrica, 68(5): 1127-1150, 2000.

[19] S. Lahaie. An analysis of alternative slot auction designs for sponsored search. In Proceedings of the 7th ACM Conference on Electronic Commerce (EC '06), pp. 218-227, 2006.

[20] S. Lahaie, D. Pennock, A. Saberi, and R. Vohra. Sponsored seach auctions. In N. Nisan, T. Roughgarden, É. Tardos, and V. V. Vazirani, editors, Algorithmic Game Theory, pp. 699-716. Cambridge University Press, 2007.

[21] N. Littlestone and M. K. Warmuth. The weighted majority algorithm. Information and Computation, 108(2): 212-261, 1994.

[22] B. Lucier. Beyond equilibria: Mechanisms for repeated combinatorial auctions. In Proceedings of the First Symposium on Innovations in Computer Science (ICS '10), pp. 166-177, 2010.

[23] B. Lucier and A. Borodin. Price of anarchy for greedy auctions. In Proceedings of the 21st Annual ACM-SIAM Symposium on Discrete Algorithms (SODA '10), pp. 537-553, 2010.

[24] B. Lucier and R. Paes Leme. GSP auctions with correlated types. In Proceedings of the 12th Annual ACM Conference on Electronic Commerce (EC'11), pp. 71-80, 2011. Preliminary version: Improved social welfare bounds for GSP at equilibrium. arXiv:1011.3268, 2010.

[25] P. Maille, E. Markakis, M. Naldi, G. D. Stamoulis, and B. Tuffin. Sponsored search auctions: An overview of research with emphasis on game theoretic aspects. Electronic Commerce Research, 12(3): 265-300, 2012.

[26] A. Mehta, A. Saberi, U. V. Vazirani, and V. V. Vazirani. Adwords and generalized online matching. Journal of the ACM, 54(5), 2007.

[27] U. Nadav and T. Roughgarden The limits of smoothness: A primal-dual framework for price of anarchy bounds. In In Proceedings of the 6th International Workshop on Internet and Network Economics (WINE'10), pp. 319-326, 2010.

[28] R. Paes Leme and É. Tardos. Sponsored search equilibria for conservative bidders. In Proceedings of the 5th Workshop on Ad Auctions (AdAuctions '09), 2009.

[29] R. Paes Leme and É. Tardos. Pure and Bayes-Nash price of anarchy for generalized second price auctions. In Proceedings of the 51st Annual IEEE Symposium on Foundations of Computer Science (FOCS '10), pp. 735-744, 2010. 
[30] P. J. Reny. On the existence of monotone pure strategy equilibria in Bayesian games. Econometrica, 79(2): 499-553, 2011.

[31] T. Roughgarden. Intrinsic robustness of the price of anarchy. In Proceedings of the 41st Annual ACM Symposium on Theory of Computing (STOC '09), pp. 513-522, 2009.

[32] T. Roughgarden. The price of anarchy in games of incomplete information. In Proceedings of the 13th ACM Conference on Electronic Commerce (EC'12), pp. 862-879, 2012.

[33] T. Roughgarden and F. Schoppmann. Local smoothness and the Price of Anarchy in atomic splittable congestion games. In Proceedings of the 22nd Annual ACM-SIAM Symposium on Discrete Algorithms (SODA '11), pp. 255-267, 2011.

[34] V. Syrgkanis. Bayesian games and the smoothness framework. arXiv:1203.5155, 2012.

[35] V. Syrgkanis and É. Tardos Composable and efficient mechanisms. In Proceedings of the 44th Symposium on Theory of Computing Conference (STOC'13), pp. 211-220, 2013. See also arXiv:1211.1325, 2012.

[36] H. R. Varian. Position auctions. International Journal of Industrial Organization, 26: 1163-1178, 2007.

\section{A Improved Bounds for Games with Uncertainty}

In this section, we prove Theorems 3.1 and 5.7 The idea of the proof is analogous to our proof of the bound of 3.164 in Section 3, based on semi-smoothness, but will use a modification of semi-smoothness specially tailored to the GSP auction game, analogous to the way we modified the simple bound of 4 derived using the $(1 / 2,1)$-semi-smoothness of GSP to a bound of 3 on the price of total anarchy for the full information case in Section 5 . We handle the case when a player has the highest effective value separately, and show that there exists a bidding profile $\mathbf{b}^{\prime}$ such that the following inequality holds.

$$
\mathbb{E}\left[\sum_{i} u_{i}\left(b_{i}^{\prime}\left(v_{i}\right), \mathbf{b}_{-i}, \gamma\right)\right] \geq \beta \mathbb{E}[O P T(\mathbf{v}, \gamma)]-(1+\delta) \sum_{i} \mathbb{E}\left[\alpha_{i} \gamma_{\pi(i)} b_{\pi(i)}\right]+\mathbb{E}\left[\alpha_{1} \gamma_{\pi(1)} b_{\pi(1)}\right]
$$

This inequality is analogous but weaker than claiming that GSP is $(\beta, \delta)$-semi-smooth, yet we will show that it implies that the price of anarchy (and the price of total anarchy) is bounded by $\frac{1+\delta}{\beta}$. This connection is stated in the next lemma.

Lemma A.1 Assume that for every GSP auction game there is a bidding profile $\mathbf{b}^{\prime}$ and parameters $\beta, \delta>0$ such that inequality (5) holds for any strategy profile $\mathbf{b}$. Then, the price of anarchy of the Generalized Second Price auction with uncertainty is at most $\frac{1+\delta}{\beta}$. The same bound applies to the price of total anarchy with uncertainty as well.

Proof. Consider a Nash equilibrium bidding profile b. Clearly, $\mathbb{E}\left[u_{i}(\mathbf{b}, \gamma)\right] \geq \mathbb{E}\left[u_{i}\left(b_{i}^{\prime}\left(v_{i}\right), \mathbf{b}_{-i}, \gamma\right)\right]$ by selecting the bidding profile $\mathbf{b}^{\prime}$ as in inequality (5). We use this inequality and the fact that the social 
welfare is the sum of the expected utilities of the advertisers plus the total payments to get

$$
\begin{aligned}
\mathbb{E}[S W(\pi(\mathbf{b}(\mathbf{v}), \gamma), \mathbf{v}, \gamma)]= & \mathbb{E}\left[\sum_{i} u_{i}(\mathbf{b}, \gamma)\right]+\mathbb{E}\left[\sum_{i} \alpha_{i} \gamma_{\pi(i+1)} b_{\pi(i+1)}\right] \\
\geq & \mathbb{E}\left[\sum_{i} u_{i}\left(b_{i}^{\prime}\left(v_{i}\right), \mathbf{b}_{-i}, \gamma\right)\right]+\mathbb{E}\left[\sum_{i} \alpha_{i} \gamma_{\pi(i+1)} b_{\pi(i+1)}\right] \\
\geq & \beta \mathbb{E}[O P T(\mathbf{v}, \gamma)]-(1+\delta) \sum_{i} \mathbb{E}\left[\alpha_{i} \gamma_{\pi(i)} b_{\pi(i)}\right]+\mathbb{E}\left[\alpha_{1} \gamma_{\pi(1)} b_{\pi(1)}\right] \\
& +\sum_{i \geq 2} \mathbb{E}\left[\alpha_{i} \gamma_{\pi(i)} b_{\pi(i)}\right] \\
= & \beta \mathbb{E}[O P T(\mathbf{v}, \gamma)]-\delta \sum_{i} \mathbb{E}\left[\alpha_{i} \gamma_{\pi(i)} b_{\pi(i)}\right] \\
\geq & \beta \mathbb{E}[O P T(\mathbf{v}, \gamma)]-\delta \mathbb{E}[S W(\pi(\mathbf{b}(\mathbf{v}), \gamma), \mathbf{v}, \gamma)],
\end{aligned}
$$

which implies that the price of anarchy is at most $\frac{1+\delta}{\beta}$, as desired. To get the same bound for the price of total anarchy, consider a coarse correlated equilibrium $\mathbf{b}$ instead of a Nash equilibrium.

The next lemma (Lemma A.3) connects inequality (5) to the existence of functions with particular properties which we call $(\beta, \delta)$-bounded functions.

Definition A.2 Let $\beta, \delta>0$ and $g:[0,1] \rightarrow \mathbb{R}_{+}$. Function $g$ is $(\beta, \delta)$-bounded if the following three properties hold:

$$
\begin{aligned}
\text { i) } \quad & \int_{0}^{1} g(y) \mathrm{d} y \leq 1, \\
\text { ii) } \quad & (1-z) \int_{z}^{1} g(y) \mathrm{d} y \geq \beta-\delta z, \quad \forall z \in[0,1], \\
\text { iii }) \quad & \int_{z}^{1}(1-y) g(y) \mathrm{d} y \geq \beta-(1+\delta) z, \quad \forall z \in[0,1] .
\end{aligned}
$$

Recall that the proof of Lemma 3.5 that GSP is $\left(1-\frac{1}{e}, 1\right)$-semi-smooth relied on a random distribution with density $f(y)=\frac{1}{1-y}$ for $y \in\left[0,\left(1-\frac{1}{e}\right)\right]$ and $f(y)=0$ otherwise, and considered the bid distribution $b_{i}^{\prime}=y v_{i}$ for player $i$ with valuation $v_{i}$. The improved proof in Lemma A.3 uses a $(\beta, \delta)$-bounded function $g$ in place of this $f$.

Lemma A.3 Let $\beta, \delta>0$ be such that a $(\beta, \delta)$-bounded function exists. Then, there is a bidding profile $\mathbf{b}^{\prime}$ such that inequality (5) holds for any strategy profile $\mathbf{b}$.

Proof. In the proof we consider a GSP auction game with $n$ slots with click-through-rates $\alpha_{1} \geq \alpha_{2} \geq \ldots \geq$ $\alpha_{n} \geq 0$ and $n$ conservative players with random valuations $v_{1}, v_{2}, \ldots, v_{n} \geq 0$ and random quality factors $\gamma_{1}, \gamma_{2}, \ldots, \gamma_{n} \geq 1$. Let $\mathbf{b}$ denote any bid profile. Also, we denote by $\mathbf{b}^{\prime}$ the bid profile such that $b_{i}^{\prime}(x)$ is the most profitable deviation for player $i$ when her valuation is $v_{i}=x$. We will prove inequality (5) using this definition for $\mathbf{b}^{\prime}$.

The proof is long and technical. Before presenting it, we give a high-level overview. We apply the following three steps: 
- Step 1: We focus on advertiser $i$ with valuation $v_{i}=x$ and obtain a lower bound on her expected utility $\mathbb{E}\left[u_{i}\left(b_{i}^{\prime}, \mathbf{b}_{-i}, \gamma\right) \mid v_{i}=x\right]$ when deviating to $b_{i}^{\prime}(x)$. The main idea we use here is that the deviation to bid $b_{i}^{\prime}(x)$ is more profitable for advertiser $i$ than deviating to the bid $y x$, for every $y \in[0,1]$. This yields infinitely many lower bounds on $\mathbb{E}\left[u_{i}\left(b_{i}^{\prime}, \mathbf{b}_{-i}, \gamma\right) \mid v_{i}=x\right]$; we combine them in a single lower bound by taking their weighted average, with weights indicated by the values of a $(\beta, \delta)$-bounded function $g$.

- Step 2: We further refine the lower bound on $\mathbb{E}\left[u_{i}\left(b_{i}^{\prime}, \mathbf{b}_{-i}, \gamma\right) \mid v_{i}=x\right]$. Here, we reason about the slots advertiser $i$ would occupy by deviating to bid $y x$ and the utility she would then have, and we use the properties of $(\beta, \delta)$-bounded functions. We consider slot 1 and slots $i \geq 2$ separately, as we did in the proof of Theorem 5.3 .

- Step 3: We use the bound obtained in Step 2 in order to compute a lower bound for the total expected utility of all players when deviating to $\mathbf{b}^{\prime}$. We first lower-bound the unconditional expected utility of advertiser $i$ and, then, we simply sum the obtained inequalities over all advertisers in order to obtain inequality (5).

Step 1: Focus on player $i$ and let $x$ be a possible valuation for this player. Let $\beta, \delta>0$ and consider a $(\beta, \delta)$-bounded function $g:[0,1] \rightarrow \mathbb{R}_{+}$. Using the first property in Definition A.2 for $g$ and the fact that $b_{i}^{\prime}(x)$ is the most profitable deviation for advertiser $i$, we have

$$
\begin{aligned}
\mathbb{E}\left[u_{i}\left(b_{i}^{\prime}(x), \mathbf{b}_{-i}, \gamma\right) \mid v_{i}=x\right] & \geq \int_{0}^{1} g(y) \mathbb{E}\left[u_{i}\left(b_{i}^{\prime}(x), \mathbf{b}_{-i}, \gamma\right) \mid v_{i}=x\right] \mathrm{d} y \\
& \geq \int_{0}^{1} g(y) \mathbb{E}\left[u_{i}\left(y x, \mathbf{b}_{-i}, \gamma\right) \mid v_{i}=x\right] \mathrm{d} y
\end{aligned}
$$

Given any slot $j$, let $A_{x}^{i j}$ denote the event that $v_{i}=x$ and $\nu(i)=j$ and $B_{x}^{i j}$ denote the event that $\nu(i)=j$ given that $v_{i}=x$. Using these definitions, we can rewrite the quantity $\mathbb{E}\left[u_{i}\left(y x, \mathbf{b}_{-i}, \gamma\right) \mid v_{i}=x\right]$ for every $y \in[0,1]$ as

$$
\mathbb{E}\left[u_{i}\left(y x, \mathbf{b}_{-i}, \gamma\right) \mid v_{i}=x\right]=\sum_{j=1}^{n} \mathbb{E}\left[u_{i}\left(y x, \mathbf{b}_{-i}, \gamma\right) \mid A_{x}^{i j}\right] \cdot \mathbb{P}\left[B_{x}^{i j}\right]
$$

By the last two (in)equalities, we obtain that

$$
\begin{aligned}
\mathbb{E}\left[u_{i}\left(b_{i}^{\prime}(x), \mathbf{b}_{-i}, \gamma\right) \mid v_{i}=x\right] & \geq \int_{0}^{1} g(y) \sum_{j=1}^{n} \mathbb{E}\left[u_{i}\left(y x, \mathbf{b}_{-i}, \gamma\right) \mid A_{x}^{i j}\right] \cdot \mathbb{P}\left[B_{x}^{i j}\right] \mathrm{d} y \\
& =\sum_{j=1}^{n} \int_{0}^{1} g(y) \mathbb{E}\left[u_{i}\left(y x, \mathbf{b}_{-i}, \gamma\right) \mid A_{x}^{i j}\right] \mathrm{d} y \cdot \mathbb{P}\left[B_{x}^{i j}\right]
\end{aligned}
$$

Step 2: Our purpose now is to refine the lower bound provided by inequality (6). Let $\pi^{i}\left(\mathbf{b}_{-i}, i\right)$ be the player with the $i$-th highest effective bid in $\mathbf{b}_{-i}$.

First consider slot 1 separately. Assume that the event $A_{x}^{i 1}$ is true, i.e., $v_{i}=x$ and $\nu(i)=1$. We will first lower-bound the quantity $\mathbb{E}\left[u_{i}\left(y x, \mathbf{b}_{-i}, \gamma\right) \mid A_{x}^{i 1}\right]$ for every $y \in[0,1]$. By deviating to bid $y x$, 
player $i$ is allocated the first slot whenever $\gamma_{i} y x>\gamma_{\pi^{i}(1)} b_{\pi^{i}(1)}$; in this case, player $i$ has utility at least $\alpha_{1}\left(\gamma_{i} x-\gamma_{\pi^{i}(1)} b_{\pi^{i}(1)}\right)$. Hence,

$$
\mathbb{E}\left[u_{i}\left(y x, \mathbf{b}_{-i}, \gamma\right) \mid A_{x}^{i 1}\right] \geq \mathbb{E}\left[\alpha_{1}\left(\gamma_{i} x-\gamma_{\pi^{i}(1)} b_{\pi^{i}(1)}\right) \mathbb{1}\left\{\gamma_{i} y x>\gamma_{\pi^{i}(1)} b_{\pi^{i}(1)}\right\} \mid A_{x}^{i 1}\right] .
$$

We set $z=\frac{\gamma_{\pi^{i}(1)} b_{\pi^{i}(1)}}{\gamma_{i} x}$ and use this last inequality to obtain

$$
\begin{aligned}
\int_{0}^{1} g(y) \mathbb{E}\left[u_{i}\left(y x, \mathbf{b}_{-i}, \gamma\right) \mid A_{x}^{i 1}\right] \mathrm{d} y & \geq \int_{0}^{1} g(y) \cdot \mathbb{E}\left[\alpha_{1} \gamma_{i} x(1-z) \mathbb{1}\{y>z\} \mid A_{x}^{i 1}\right] \mathrm{d} y \\
& =\mathbb{E}\left[\alpha_{1} \gamma_{i} x(1-z) \int_{0}^{1} g(y) \mathbb{1}\{y>z\} \mathrm{d} y \mid A_{x}^{i 1}\right] \\
& =\mathbb{E}\left[\alpha_{1} \gamma_{i} x(1-z) \int_{z}^{1} g(y) \mathrm{d} y \mid A_{x}^{i 1}\right] \\
& \geq \mathbb{E}\left[\alpha_{1}\left(\beta \gamma_{i} x-\delta \gamma_{\pi^{i}(1)} b_{\pi^{i}(1)}\right) \mid A_{x}^{i 1}\right]
\end{aligned}
$$

where the second inequality follows by the second property of Definition A.2 for function $g$ (and using the definition of $z$ ).

Now, assume that the event $A_{x}^{i j}$ is true for $j \geq 2$, i.e., $v_{i}=x$ and $\nu(i)=j$. We will lower-bound the quantity $\mathbb{E}\left[u_{i}\left(y x, \mathbf{b}_{-i}, \gamma\right) \mid A_{x}^{i j}\right]$ for every $y \in[0,1]$. By deviating to bid $y x$, player $i$ is allocated slot $j$ (or a higher one) whenever $\gamma_{i} y x>\gamma_{\pi^{i}(j)} b_{\pi^{i}(j)}$; in this case, player $i$ has utility at least $\alpha_{j} \gamma_{i} x(1-y)$. Hence,

$$
\mathbb{E}\left[u_{i}\left(y x, \mathbf{b}_{-i}, \gamma\right) \mid A_{x}^{i j}\right] \geq \mathbb{E}\left[\alpha_{j} \gamma_{i} x(1-y) \mathbb{1}\left\{\gamma_{i} y x>\gamma_{\pi^{i}(j)} b_{\pi^{i}(j)}\right\} \mid A_{x}^{i j}\right]
$$

We set $z=\frac{\gamma_{\pi^{i}(j)} b_{\pi^{i}(j)}}{\gamma_{i} x}$ and use this last inequality to obtain

$$
\begin{aligned}
\int_{0}^{1} g(y) \mathbb{E}\left[u_{i}\left(y x, \mathbf{b}_{-i}, \gamma\right) \mid A_{x}^{i j}\right] \mathrm{d} y & \geq \int_{0}^{1} g(y) \mathbb{E}\left[\alpha_{j} \gamma_{i} x(1-y) \mathbb{1}\{y>z\} \mid A_{x}^{i j}\right] \mathrm{d} y \\
& =\mathbb{E}\left[\alpha_{j} \gamma_{i} x \int_{z}^{1}(1-y) g(y) \mathrm{d} y \mid A_{x}^{i j}\right] \\
& \geq \mathbb{E}\left[\alpha_{j}\left(\beta \gamma_{i} x-(1+\delta) \gamma_{\pi^{i}(j)} b_{\pi^{i}(j)}\right) \mid A_{x}^{i j}\right] .
\end{aligned}
$$

The second inequality follows by the third property of Definition A.2 for function $g$ (and using the definition of $z$ ).

We now use inequality (6) together with the lower bounds for $\int_{0}^{1} g(y) \mathbb{E}\left[u_{i}\left(y x, \mathbf{b}_{-i}, \gamma\right) \mid A_{x}^{i j}\right] \mathrm{d} y$ obtained in (7) and (8). We have

$$
\begin{aligned}
\mathbb{E}\left[u_{i}\left(b_{i}^{\prime}(x), \mathbf{b}_{-i}, \gamma\right) \mid v_{i}=x\right] \geq & \mathbb{E}\left[\alpha_{1}\left(\beta \gamma_{i} x-\delta \gamma_{\pi^{i}(1)} b_{\pi^{i}(1)}\right) \mid A_{x}^{i 1}\right] \cdot \mathbb{P}\left[B_{x}^{i 1}\right] \\
& +\sum_{j=2}^{n} \mathbb{E}\left[\alpha_{j}\left(\beta \gamma_{i} x-(1+\delta) \gamma_{\pi^{i}(j)} b_{\pi^{i}(j)}\right) \mid A_{x}^{i j}\right] \cdot \mathbb{P}\left[B_{x}^{i j}\right] \\
= & \beta \sum_{j=1}^{n} \mathbb{E}\left[\alpha_{j} \gamma_{i} x \mid A_{x}^{i j}\right] \cdot \mathbb{P}\left[B_{x}^{i j}\right]-\delta \mathbb{E}\left[\alpha_{1} \gamma_{\pi^{i}(1)} b_{\pi^{i}(1)} \mid A_{x}^{i 1}\right] \cdot \mathbb{P}\left[B_{x}^{i 1}\right] \\
& -(1+\delta) \sum_{j=2}^{n} \mathbb{E}\left[\alpha_{j} \gamma_{\pi^{i}(j)} b_{\pi^{i}(j)} \mid A_{x}^{i j}\right] \cdot \mathbb{P}\left[B_{x}^{i j}\right] .
\end{aligned}
$$


Step 3: We can now bound the unconditional expected utility of player $i$ when deviating to strategy $b_{i}^{\prime}\left(v_{i}\right)$ by integrating over the range of valuations for player $i$ and using the last inequality obtained in Step 2. In the following we use $f_{v_{i}}(x)$ to denote the probability density function of the random variable $v_{i}$. We have

$$
\begin{aligned}
\mathbb{E}\left[u_{i}\left(b_{i}^{\prime}\left(v_{i}\right), \mathbf{b}_{-i}, \gamma\right)\right]= & \int_{0}^{\infty} \mathbb{E}\left[u_{i}\left(b_{i}^{\prime}\left(v_{i}\right), \mathbf{b}_{-i}, \gamma\right)\right] \cdot f_{v_{i}}(x) \mathrm{d} x \\
\geq & \beta \sum_{j=1}^{n} \int_{0}^{\infty} \mathbb{E}\left[\alpha_{j} \gamma_{i} v_{i} \mid A_{x}^{i j}\right] \cdot \mathbb{P}\left[B_{x}^{i j}\right] \cdot f_{v_{i}}(x) \mathrm{d} x \\
& -\delta \int_{0}^{\infty} \mathbb{E}\left[\alpha_{1} \gamma_{\pi^{i}(1)} b_{\pi^{i}(1)} \mid A_{x}^{i 1}\right] \cdot \mathbb{P}\left[B_{x}^{i 1}\right] \cdot f_{v_{i}}(x) \mathrm{d} x \\
& -(1+\delta) \sum_{j=2}^{n} \int_{0}^{\infty} \mathbb{E}\left[\alpha_{j} \gamma_{\pi^{i}(j)} b_{\pi^{i}(j)} \mid A_{x}^{i j}\right] \cdot \mathbb{P}\left[B_{x}^{i j}\right] \cdot f_{v_{i}}(x) \mathrm{d} x .
\end{aligned}
$$

Now, we use the property

$$
\int_{0}^{\infty} \mathbb{E}\left[Z \mid A_{x}^{i j}\right] \cdot \mathbb{P}\left[B_{x}^{i j}\right] \cdot f_{v_{i}}(x) \mathrm{d} x=\mathbb{E}[Z \mid \nu(i)=j] \cdot \mathbb{P}[\nu(i)=j],
$$

for any random variable $Z$ as well as the fact that $\gamma_{\pi^{i}(j)} b_{\pi^{i}(j)} \leq \gamma_{\pi(j)} b_{\pi(j)}$ to obtain that

$$
\begin{aligned}
& \mathbb{E}\left[u_{i}\left(b_{i}^{\prime}\left(v_{i}\right), \mathbf{b}_{-i}, \gamma\right)\right] \\
\geq & \beta \sum_{j=1}^{n} \mathbb{E}\left[\alpha_{j} \gamma_{i} v_{i} \mid \nu(i)=j\right] \cdot \mathbb{P}[\nu(i)=j]-\delta \mathbb{E}\left[\alpha_{1} \gamma_{\pi^{i}(1)} b_{\pi^{i}(1)} \mid \nu(i)=1\right] \cdot \mathbb{P}[\nu(i)=1] \\
& -(1+\delta) \sum_{j=2}^{n} \mathbb{E}\left[\alpha_{j} \gamma_{\pi^{i}(j)} b_{\pi^{i}(j)} \mid \nu(i)=j\right] \cdot \mathbb{P}[\nu(i)=j] \\
\geq & \beta \sum_{j=1}^{n} \mathbb{E}\left[\alpha_{j} \gamma_{i} v_{i} \mid \nu(i)=j\right] \cdot \mathbb{P}[\nu(i)=j]-\delta \mathbb{E}\left[\alpha_{1} \gamma_{\pi(1)} b_{\pi(1)} \mid \nu(i)=1\right] \cdot \mathbb{P}[\nu(i)=1] \\
& -(1+\delta) \sum_{j=2}^{n} \mathbb{E}\left[\alpha_{j} \gamma_{\pi(j)} b_{\pi(j)} \mid \nu(i)=j\right] \cdot \mathbb{P}[\nu(i)=j] \\
= & \beta \sum_{j=1}^{n} \mathbb{E}\left[\alpha_{j} \gamma_{i} v_{i} \mid \nu(i)=j\right] \cdot \mathbb{P}[\nu(i)=j]-(1+\delta) \sum_{j=1}^{n} \mathbb{E}\left[\alpha_{j} \gamma_{\pi(j)} b_{\pi(j)} \mid \nu(i)=j\right] \cdot \mathbb{P}[\nu(i)=j] \\
& +\mathbb{E}\left[\alpha_{1} \gamma_{\pi(1)} b_{\pi(1)} \mid \nu(i)=1\right] \cdot \mathbb{P}[\nu(i)=1] \\
= & \beta \mathbb{E}\left[\alpha_{\nu(i)} \gamma_{i} v_{i}\right]-(1+\delta) \mathbb{E}\left[\alpha_{\nu(i)} \gamma_{\pi(\nu(i))} b_{\pi(\nu(i))}\right]+\mathbb{E}\left[\alpha_{1} \gamma_{\pi(1)} b_{\pi(1)} \mid \nu(i)=1\right] \cdot \mathbb{P}[\nu(i)=1] .
\end{aligned}
$$

By summing over all players, we obtain inequality (5). In particular,

$$
\begin{aligned}
\sum_{i} \mathbb{E}\left[u_{i}\left(b_{i}^{\prime}\left(v_{i}\right), \mathbf{b}_{-i}, \gamma\right)\right] \geq & \beta \sum_{i} \mathbb{E}\left[\alpha_{\nu(i)} \gamma_{i} v_{i}\right]-(1+\delta) \sum_{i} \mathbb{E}\left[\alpha_{\nu(i)} \gamma_{\pi(\nu(i))} b_{\pi(\nu(i))}\right] \\
& +\sum_{i} \mathbb{E}\left[\alpha_{1} \gamma_{\pi(1)} b_{\pi(1)} \mid \nu(i)=1\right] \cdot \mathbb{P}[\nu(i)=1] \\
= & \beta \mathbb{E}[O P T(\mathbf{v}, \gamma)]-(1+\delta) \sum_{i} \mathbb{E}\left[\alpha_{i} \gamma_{\pi(i)} b_{\pi(i)}\right]+\mathbb{E}\left[\alpha_{1} \gamma_{\pi(1)} b_{\pi(1)}\right] .
\end{aligned}
$$


Therefore, by Lemmas A.1 and A.3, in order to bound the price of anarchy, it suffices to find a $(\beta, \delta)$ bounded function such that the ratio $\frac{1+\delta}{\beta}$ is as low as possible. This is the purpose of the following lemma.

Lemma A.4 Consider a function $g:[0,1] \rightarrow \mathbb{R}_{+}$defined as follows:

$$
g(y)= \begin{cases}\frac{\kappa}{1-y}, & y \in[0, \lambda) \\ \frac{(\kappa-1)(1-\mu)}{(1-y)^{2}}, & y \in[\lambda, \mu) \\ 0, & y \in[\mu, 1]\end{cases}
$$

where $\kappa>1$ and $1>\mu \geq \lambda \geq 0$ such that $\frac{(\kappa-1)(\mu-\lambda)}{1-\lambda}-\kappa \ln (1-\lambda) \leq 1$, and $(\kappa-1)(1-\mu) \ln \frac{1-\lambda}{1-\mu}-$ $(\kappa-1) \mu+\kappa \lambda \geq 0$. Then, $g(y)$ is an $((\kappa-1) \mu, \kappa-1)$-bounded function.

Proof. We begin by computing $\int_{0}^{1} g(y) \mathrm{d} y$. It holds that

$$
\int_{0}^{1} g(y) \mathrm{d} y=\int_{0}^{\lambda} \frac{\kappa}{1-y} \mathrm{~d} y+\int_{\lambda}^{\mu} \frac{(\kappa-1)(1-\mu)}{(1-y)^{2}} \mathrm{~d} y=\frac{(\kappa-1)(\mu-\lambda)}{1-\lambda}-\kappa \ln (1-\lambda) \leq 1,
$$

where the inequality holds by the first assumption concerning $\kappa, \lambda$ and $\mu$. Hence, $g$ satisfies the first property of Definition A.2.

For the second property of Definition A.2 it suffices to prove that

$$
(1-z) \int_{z}^{1} g(y) \mathrm{d} y+(\kappa-1)(z-\mu) \geq 0, \quad \forall z \in[0,1] .
$$

We distinguish between three cases depending on $z$. First, we consider the case that $z \in[\mu, 1]$. We have

$$
(1-z) \int_{z}^{1} g(y) \mathrm{d} y+(\kappa-1)(z-\mu)=(\kappa-1)(z-\mu) \geq 0
$$

where the inequality holds since $z \in[\mu, 1]$ and $\kappa>1$. For $z \in(\lambda, \mu)$ we have

$$
(1-z) \int_{z}^{1} g(y) \mathrm{d} y+(\kappa-1)(z-\mu)=(1-z) \int_{z}^{\mu} \frac{(\kappa-1)(1-\mu)}{(1-y)^{2}} \mathrm{~d} y+(\kappa-1)(z-\mu)=0 .
$$

Finally, for $z \in[0, \lambda]$ we have

$$
\begin{aligned}
& (1-z) \int_{z}^{1} g(y) \mathrm{d} y+(\kappa-1)(z-\mu) \\
= & (1-z) \int_{z}^{\lambda} \frac{\kappa}{1-y} \mathrm{~d} y+(1-z) \int_{\lambda}^{\mu} \frac{(\kappa-1)(1-\mu)}{(1-y)^{2}} \mathrm{~d} y+(\kappa-1)(z-\mu) \\
= & (1-z) \kappa \ln \frac{1-z}{1-\lambda}+\frac{(1-z)(\kappa-1)(\mu-\lambda)}{1-\lambda}+(\kappa-1)(z-\mu) \\
\geq & (\kappa-1)(\mu-\lambda)+(\kappa-1)(\lambda-\mu) \\
= & 0,
\end{aligned}
$$


where the inequality follows by the fact that the derivative with respect to $z$ is negative for $z \in[0, \lambda]$. Hence, it holds that $g$ satisfies the second property of Definition A.2

It remains to prove that $g$ satisfies the third property of Definition A.2. Similarly, it suffices to prove that

$$
\int_{z}^{1}(1-y) g(y) \mathrm{d} y-(\kappa-1) \mu+\kappa z \geq 0, \quad \forall z \in[0,1] .
$$

Again, we distinguish between three cases depending on $z$. First, we consider the case that $z \in[\mu, 1]$. We have

$$
\int_{z}^{1}(1-y) g(y) \mathrm{d} y-(\kappa-1) \mu+\kappa z=-(\kappa-1) \mu+\kappa z \geq \mu \geq 0,
$$

where the first inequality follows since $z \in[\mu, 1]$. For $z \in[\lambda, \mu)$ we have

$$
\begin{aligned}
\int_{z}^{1}(1-y) g(y) \mathrm{d} y-(\kappa-1) \mu+\kappa z & =\int_{z}^{\mu} \frac{(\kappa-1)(1-\mu)}{(1-y)^{2}} \mathrm{~d} y-(\kappa-1) \mu+\kappa z \\
& =(\kappa-1)(1-\mu) \ln \frac{1-z}{1-\mu}-(\kappa-1) \mu+\kappa z \\
& \geq(\kappa-1)(1-\mu) \ln \frac{1-\lambda}{1-\mu}-(\kappa-1) \mu+\kappa \lambda \\
& \geq 0
\end{aligned}
$$

where the first inequality follows by the fact that the derivative with respect to $z$ is strictly positive for $z \in[\lambda, \mu)$, and the second inequality follows by the second assumption concerning $\kappa, \lambda$ and $\mu$. Finally, for $z \in[0, \lambda)$ we have

$$
\begin{aligned}
\int_{z}^{1}(1-y) g(y) \mathrm{d} y-(\kappa-1) \mu+\kappa z & =\int_{z}^{\lambda} \kappa \mathrm{d} y+\int_{\lambda}^{\mu} \frac{(\kappa-1)(1-\mu)}{(1-y)^{2}} \mathrm{~d} y-(\kappa-1) \mu+\kappa z \\
& =(\kappa-1)(1-\mu) \ln \frac{1-\lambda}{1-\mu}-(\kappa-1) \mu+\kappa \lambda \\
& \geq 0,
\end{aligned}
$$

where the inequality follows by the second assumption concerning $\kappa, \lambda$ and $\mu$. The proof of the lemma is complete.

We are now ready to complete the proof of Theorems 3.1 and 5.7. The two conditions of Lemma A.4 are satisfied for $\kappa=1.7507, \lambda=0.225$, and $\mu=0.7966$. By combining Lemmas A.1, A.3, and A.4 we conclude that the price of (total) anarchy of GSP auction games over Bayes-Nash equilibria is at most $\frac{\kappa}{(\kappa-1) \mu}<2.9276$.

\section{B Improved Bounds for Pure Nash Equilibria}

In this section we present our results for pure Nash equilibria in the full information setting (Theorems 4.1 and 4.2). For simplicity of exposition, we consider all quality factors to be equal to 1 ; so, $\gamma$ does not appear in notation. Our proofs can be adapted to different quality factors in a straightforward way. We consider GSP auction games with $n$ advertisers with valuations $v_{1} \geq \ldots \geq v_{n} \geq 0$ and $n$ slots with click-through-rates 
$\alpha_{1} \geq \ldots \geq \alpha_{n} \geq 0$. We assume that neither all slots have the same click-through-rate nor all advertisers have the same valuation (in both cases, the price of anarchy is 1).

We use the term inefficiency of allocation $\pi$ to refer to the ratio $O P T(\mathbf{v}) / S W(\pi, \mathbf{v})$. In Lemma 4.5 we showed that every pure Nash equilibrium corresponds to a weakly feasible allocation. Hence, the price of anarchy of a GSP auction game over pure Nash equilibria is upper-bounded by the worst-case inefficiency among weakly feasible allocations.

Definition B.1 An allocation $\pi$ is called proper if for any two slots $i<j$ with equal click-through-rates, it holds $\pi(i)<\pi(j)$.

Clearly, for any non-proper weakly feasible allocation, we can construct a proper weakly feasible one with equal social welfare. Hence, in order to prove our upper bounds, we essentially upper-bound the worst-case inefficiency over proper weakly feasible allocations.

Given an allocation $\pi$, consider the directed graph $G(\pi)$ that has one node for each slot, and a directed edge for each advertiser $i$ that connects the node corresponding to slot $i$ to the node corresponding to slot $\pi^{-1}(i)$. In general, $G(\pi)$ consists of a set of disjoint cycles and may contain self-loops.

Definition B.2 An allocation $\pi$ is called reducible if its directed graph $G(\pi)$ has more than one cycles. Otherwise, it is called irreducible.

Given a reducible allocation $\pi$ such that $G(\pi)$ has $c \geq 2$ cycles, we can construct $c$ GSP auction subgames by considering the slots and the advertisers that correspond to the nodes and edges of each cycle. Similarly, for $\ell=1, \ldots, c$, the restriction $\pi^{\ell}$ of $\pi$ to the slots and advertisers of the $\ell$-th subgame is an allocation for this game. The next fact essentially states that we can focus on irreducible allocations.

Fact B.3 If allocation $\pi$ is weakly feasible for the original GSP auction game, then $\pi^{\ell}$ is weakly feasible for the $\ell$-th subgame as well, for $\ell=1, \ldots, c$. Then, the inefficiency of $\pi$ is at most the maximum inefficiency among the allocations $\pi^{\ell}$ for $\ell=1, \ldots, c$.

When considering irreducible weakly feasible allocations, we further assume that the index of the slot advertiser 1 occupies is smaller than the index of the advertiser that is assigned to slot 1 . This is without loss of generality due to the following argument. Consider an irreducible weakly feasible allocation $\pi$ for a GSP auction game with $n$ advertisers such that $\pi^{-1}(1)>\pi(1)$. We construct a new game with clickthrough-rate $a_{i}^{\prime}=v_{i}$ for slot $i$ and valuation $v_{i}^{\prime}=\alpha_{i}$ for advertiser $i$, for $i=1, \ldots, n$, and the allocation $\pi_{*}=\pi^{-1}$. Observe that $\pi_{*}^{-1}(1)=\pi(1)<\pi^{-1}(1)=\pi_{*}(1)$. Clearly, the optimal social welfare is the same in both games while the social welfare of $\pi_{*}$ for the new game is $S W\left(\pi_{*}, \mathbf{v}^{\prime}\right)=\sum_{i} a_{i}^{\prime} v_{\pi_{*}(i)}^{\prime}=$ $\sum_{i} v_{i} \alpha_{\pi_{*}(i)}=\sum_{i} \alpha_{\pi^{-1}(i)} v_{i}=S W(\pi, \mathbf{v})$. We can also prove the weak feasibility conditions for $\pi_{*}$ in the new game for each $i, j$. In order to do so, consider the weak feasibility condition for $\pi$ in the original game for advertisers $\pi(j), \pi(i)$. It is $\alpha_{j} v_{\pi(j)} \geq \alpha_{i}\left(v_{\pi(j)}-v_{\pi(i)}\right)$ and, equivalently, $v_{\pi(i)} \alpha_{i} \geq v_{\pi(j)}\left(\alpha_{i}-\alpha_{j}\right)$. By the definition of the click-through-rates and the valuations in the new game and the definition of $\pi_{*}$, we obtain that $a_{\pi_{*}^{-1}(i)}^{\prime} v_{i}^{\prime} \geq a_{\pi_{*}^{-1}(j)}^{\prime}\left(v_{i}^{\prime}-v_{j}^{\prime}\right)$ as desired.

We furthermore note that when $v_{n}=0$, any proper weakly feasible allocation is reducible. This is obviously the case if all advertisers with zero valuation use the last slots. Otherwise, consider an advertiser $i$ with non-zero valuation that is assigned a slot $\pi^{-1}(i)>\pi^{-1}(j)$ where $j$ is an advertiser with zero valuation. Since the allocation is proper, it holds that $\alpha_{\pi^{-1}(i)}<\alpha_{\pi^{-1}(j)}$. Then, we obtain a contradiction by the weak feasibility condition $\alpha_{\pi^{-1}(i)} v_{i} \geq \alpha_{\pi^{-1}(j)}\left(v_{i}-v_{j}\right)$ for advertisers $i, j$. 


\section{B.1 GSP auction games with two and three advertisers}

We now complete the proof of Theorem 4.2 .

We begin by presenting the matching upper bound on the price of anarchy for two advertisers and two slots. The upper bound follows by bounding the inefficiency of weakly feasible allocations. Consider a GSP auction game with two slots with click-through-rates $\alpha_{1} \geq \alpha_{2}=\beta \alpha_{1}$, for $\beta \in[0,1]$ and two advertisers with valuations $v_{1} \geq v_{2}=\lambda v_{1}$, for $\lambda \in[0,1]$. The only non-optimal weakly feasible allocation $\pi$ assigns advertiser 1 to slot 2 and advertiser 2 to slot 1 . Its social welfare is $S W(\pi, \mathbf{v})=\alpha_{1} v_{2}+\alpha_{2} v_{1}=\alpha_{1} v_{1}(\beta+\lambda)$, while the optimal social welfare is $\operatorname{OPT}(\mathbf{v})=\alpha_{1} v_{1}+\alpha_{2} v_{2}=\alpha_{1} v_{1}(1+\beta \lambda)$. Furthermore, the weak feasibility condition for advertiser 1 implies that $\alpha_{2} v_{1} \geq \alpha_{1}\left(v_{1}-v_{2}\right)$, i.e., $\beta \geq 1-\lambda$. We have that

$$
\frac{O P T(\mathbf{v})}{S W(\pi, \mathbf{v})}=\frac{1+\beta \lambda}{\beta+\lambda} \leq \frac{1+(\beta+\lambda)^{2} / 4}{\beta+\lambda} \leq 5 / 4
$$

where the first inequality holds since the product $\beta \lambda$ is maximized when $\beta=\lambda=(\beta+\lambda) / 2$ and the second inequality holds since $\beta+\lambda \in[1,2]$ and the function $\frac{1+x^{2} / 4}{x}$ is non-increasing in $x \in[1,2]$.

For the case of three advertisers, we again present a tight bound on the price of anarchy. We first present the upper bound. Consider a GSP auction game with three slots with click-through-rates $\alpha_{1} \geq \alpha_{2} \geq \alpha_{3} \geq 0$ and three advertisers with valuations $v_{1} \geq v_{2} \geq v_{3} \geq 0$ and a proper weakly feasible allocation $\pi$ of slots to advertisers. We will prove the theorem by upper-bounding the inefficiency of $\pi$ by 1.259134. If $\pi$ is reducible, then the inefficiency is bounded by the inefficiency of games with two advertisers (see Fact B.3) and the theorem follows by the upper bound of $5 / 4$ proved for this case. So, in the following, we assume that $\pi$ is irreducible; by the observation above, this implies that $v_{3}>0$. There are only two such allocations which are in fact symmetric: in the first, slots $1,2,3$ are allocated to advertisers $3,1,2$, respectively, and in the second, slots 1, 2, 3 are allocated to advertisers 2, 3, 1, respectively. Without loss of generality (see the discussion above), we assume that $\pi$ is the former allocation.

Let $\beta, \delta, \lambda$, and $\mu$ be such that $\alpha_{2}=\beta \alpha_{1}, \alpha_{3}=\delta \alpha_{1}, v_{2}=\lambda v_{1}$, and $v_{3}=\mu v_{1}$. Clearly, it holds that $1 \geq$ $\beta \geq \delta \geq 0$ and $1 \geq \lambda \geq \mu>0$. The social welfare of allocation $\pi$ is $S W(\pi, \mathbf{v})=\alpha_{1} v_{1}(\mu+\beta+\delta \lambda)$ whereas the optimal social welfare is $O P T(\mathbf{v})=\alpha_{1} v_{1}(1+\beta \lambda+\delta \mu)$. Furthermore, since $\pi$ is weakly feasible, the weak feasibility conditions for advertisers 1 and 3 and advertisers 2 and 3 are $\alpha_{2} v_{1} \geq \alpha_{1}\left(v_{1}-v_{3}\right)$ and $\alpha_{3} v_{2} \geq \alpha_{1}\left(v_{2}-v_{3}\right)$, respectively, i.e., $\beta \geq 1-\mu$ and $\delta \geq 1-\frac{\mu}{\lambda}$. We are now ready to bound the inefficiency of $\pi$. Let $\epsilon, \theta \geq 0$ be such that $\beta=1-\mu+\epsilon$ and $\delta=1-\frac{\mu}{\lambda}+\theta$. We have

$$
\begin{aligned}
\frac{O P T(\mathbf{v})}{S W(\pi, \mathbf{v})} & =\frac{1+\beta \lambda+\delta \mu}{\mu+\beta+\delta \lambda}=\frac{1+\lambda-\mu \lambda+\mu-\frac{\mu^{2}}{\lambda}+\epsilon \lambda+\theta \mu}{1+\lambda-\mu+\epsilon+\theta \lambda} \\
& \leq \frac{1+\lambda-\mu \lambda+\mu-\frac{\mu^{2}}{\lambda}}{1+\lambda-\mu} .
\end{aligned}
$$

The inequality follows since $1 \geq \lambda \geq \mu>0$ implies that $1+\lambda-\mu \lambda+\mu-\frac{\mu^{2}}{\lambda}=1+\lambda-\mu+\mu(1-\lambda)+$ $\mu(1-\mu / \lambda) \geq 1+\lambda-\mu \geq 1$ and $\epsilon+\theta \lambda \geq \epsilon \lambda+\theta \mu \geq 0$.

For $\mu \in[0,1]$, this last expression is maximized for the value of $\mu$ that makes its derivative with respect to $\mu$ equal to zero, i.e., $\mu=-\sqrt{\lambda^{3}+1}+\lambda+1$. By substituting $\mu$, we obtain that

$$
\frac{O P T(\mathbf{v})}{S W(\pi, \mathbf{v})} \leq \frac{\lambda^{2}+\lambda+2-2 \sqrt{\lambda^{3}+1}}{\lambda} \leq 1+2 \zeta=1.259134
$$

where $\zeta=0.129567$ and the second inequality follows by the following lemma (Lemma B.4). 
Lemma B.4 Let $\zeta=0.129567$. For any $\lambda \in[0,1]$, it holds that $\sqrt{\lambda^{3}+1} \geq 1-\zeta \lambda+\frac{\lambda^{2}}{2}$.

Proof. Since both parts of the inequality are non-negative for $\lambda \in[0,1]$, it suffices to show that the function $f(\lambda)=\left(\lambda^{3}+1\right)-\left(1-\zeta \lambda+\frac{\lambda^{2}}{2}\right)^{2}$ is non-negative for $\lambda \in[0,1]$. Let $g(\lambda)=-\frac{\lambda^{3}}{4}+(1+\zeta) \lambda^{2}-(1+$ $\left.\zeta^{2}\right) \lambda+2 \zeta$ and observe that $f(\lambda)=\lambda \cdot g(\lambda)$. The proof will follow by proving that $g(\lambda) \geq 0$ when $\lambda \in[0,1]$. Observe that the derivative of $g$ is strictly negative for $\lambda=0$ and strictly positive for $\lambda=1$. Hence, the minimum of $g$ in $[0,1]$ is achieved at the point $\lambda^{*}=\frac{4+4 \zeta-2 \sqrt{\zeta^{2}+8 \zeta+1}}{3}$ where the derivative of $g$ becomes zero. Straightforward calculations yield that $g\left(\lambda^{*}\right)>0$ and the lemma follows.

In the following we prove that the above analysis is tight. Consider a GSP auction game with three advertisers with valuations $v_{1}=1, v_{2}=0.5296$, and $v_{3}=0.14583$, respectively, and three slots with click-through-rates $\alpha_{1}=1, \alpha_{2}=0.55071$, and $\alpha_{3}=0.4704$, respectively. Let $\mathbf{b}=\left(b_{1}, b_{2}, b_{3}\right)$ be a bid vector with $b_{1}=0, b_{2}=v_{2}=0.5296$, and $b_{3}=v_{3}=0.14583$, respectively. So, advertiser 2 is allocated slot 1 , advertiser 3 is allocated slot 2 , and advertiser 1 is allocated slot 3 . We refer to this allocation as $\pi$. It is not hard to verify that $\mathbf{b}$ is a pure Nash equilibrium, and that the price of anarchy is given by:

$$
\frac{O P T(\mathbf{v})}{S W(\pi, \mathbf{v})}=\frac{\alpha_{1} v_{1}+\alpha_{2} v_{2}+\alpha_{3} v_{3}}{\alpha_{1} v_{2}+\alpha_{2} v_{3}+\alpha_{3} v_{1}} \geq 1.259133
$$

The proof of Theorem 4.2 is complete.

\section{B.2 GSP auction games with many advertisers}

We now prove Theorem 4.1 In order to do so, we will actually prove the stronger claim that the worstcase inefficiency among weakly feasible allocations of any GSP auction game is at most $r=\frac{61+7 \sqrt{217}}{128} \approx$ 1.28216. We use induction. As the base of our induction, we use the fact that GSP auction games with one, two, or three advertisers have worst-case inefficiency among weakly feasible allocations at most 1.28216. For a single advertiser, the claim is trivial. For two or three advertisers, it follows by the proof of Theorem 4.2. Let $n \geq 4$ be an integer. Using the inductive hypothesis that the worst-case inefficiency among weakly feasible allocations of any GSP auction game with at most $n-1$ advertisers is at most $r$, we will show that this is also the case for any GSP auction game with $n$ advertisers.

Consider a GSP auction game with $n$ advertisers with valuations $v_{1} \geq v_{2} \geq \ldots \geq v_{n} \geq 0$ and $n$ slots with click-through-rates $\alpha_{1} \geq \alpha_{2} \geq \ldots \geq \alpha_{n} \geq 0$ and let $\pi$ be a proper weakly feasible allocation. If $\pi$ is reducible, the claim follows by Fact B.3 and the inductive hypothesis. So, in the following, we assume that $\pi$ is irreducible; this implies that $v_{n}>0$. Let $j$ be the advertiser that is assigned slot 1 and $i_{1}$ be the slot assigned to advertiser 1 . Without loss of generality, we assume that $i_{1}<j$ since the other case is symmetric; see the discussion at the beginning of Section $\mathrm{B}$ Also, let $i_{2}$ be the slot assigned to advertiser $i_{1}$. By our assumptions, the integers $j, 1, i_{1}$, and $i_{2}$ are different.

We will show that

$$
S W(\pi, \mathbf{v}) \geq \alpha_{1} v_{j}+\alpha_{i_{1}}\left(v_{1}-\frac{v_{i_{1}}}{r}\right)+\alpha_{i_{2}}\left(v_{i_{1}}-v_{j}\right)-\frac{\alpha_{1} v_{1}}{r}+\frac{O P T(\mathbf{v})}{r} .
$$

Once we have proved inequality (9), we can obtain the desired relation between $S W(\pi, \mathbf{v})$ and $O P T(\mathbf{v})$ using the following technical lemma. 
Lemma B.5 Let $r=\frac{61+7 \sqrt{217}}{128} \approx 1.28216$ and $f(\beta, \delta, \lambda, \mu)=\mu+\beta\left(1-\frac{\lambda}{r}\right)+\delta(\lambda-\mu)-\frac{1}{r}$. Then, the objective value of the mathematical program

$$
\begin{aligned}
\text { minimize } & f(\beta, \delta, \lambda, \mu) \\
\text { subject to } & \beta \geq 1-\mu \\
& \delta \geq 1-\mu / \lambda \\
& 1 \geq \lambda \geq \mu>0 \\
& 1 \geq \beta, \delta \geq 0
\end{aligned}
$$

is non-negative.

Proof. Since $\mu \leq \lambda \leq 1$, we have that $f(\beta, \delta, \lambda, \mu)$ is non-decreasing in $\beta$ and $\delta$. Using the first two constraints, we have that the objective value of the mathematical program is at least

$$
f\left(1-\mu, 1-\frac{\mu}{\lambda}, \lambda, \mu\right)=1-\frac{1}{r}+\lambda-\frac{\lambda}{r}-\mu\left(2-\frac{\lambda}{r}\right)+\frac{\mu^{2}}{\lambda}
$$

which is minimized for $\mu=\lambda-\frac{\lambda^{2}}{2 r}$ to

$$
f\left(1-\lambda+\frac{\lambda^{2}}{2 r}, \frac{\lambda}{2 r}, \lambda, \lambda-\frac{\lambda^{2}}{2 r}\right)=1-\frac{1}{r}-\frac{\lambda}{r}+\frac{\lambda^{2}}{r}-\frac{\lambda^{3}}{4 r^{2}} .
$$

In order to complete the proof it suffices to show that the function $g(\lambda)=1-\frac{1}{r}-\frac{\lambda}{r}+\frac{\lambda^{2}}{r}-\frac{\lambda^{3}}{4 r^{2}}$ is nonnegative for $\lambda \in[0,1]$. Observe that $g(\lambda)$ is a polynomial of degree 3 and, hence, it has at most one local minimum. Also observe that the derivative of $g(\lambda)$ is $-\frac{1}{r}+\frac{2 \lambda}{r}-\frac{3 \lambda^{2}}{4 r^{2}}$ which is strictly negative for $\lambda=0$ and strictly positive for $\lambda=1$. Hence, its minimum in $[0,1]$ is achieved at the point $\lambda^{*}=\frac{4 r-2 \sqrt{4 r^{2}-3 r}}{3}$ where the derivative becomes zero. Straightforward calculations yield that $g\left(\lambda^{*}\right)=0$ and the lemma follows.

So, assuming that (9) holds, we can apply Lemma B.5 with $\beta=\alpha_{i_{1}} / \alpha_{1}, \delta=\alpha_{i_{2}} / \alpha_{1}, \lambda=v_{i_{1}} / v_{1}$, and $\mu=v_{j} / v_{1}$. Clearly, the last two constraints of the mathematical program in LemmaB.5are satisfied. Also, observe that the weak feasibility conditions for advertisers 1 and $j$ and advertisers $i_{1}$ and $j$ in allocation $\pi$ are $\alpha_{i_{1}} v_{1} \geq \alpha_{1}\left(v_{1}-v_{j}\right)$ and $\alpha_{i_{2}} v_{i_{1}} \geq \alpha_{1}\left(v_{i_{1}}-v_{j}\right)$, respectively, i.e., $\beta \geq 1-\mu$ and $\delta \geq 1-\mu / \lambda$ and the first two constraints of the mathematical program in Lemma B.5 are satisfied as well. Now, using inequality (9) and Lemma B.5, we have that

$$
S W(\pi, \mathbf{v}) \geq f\left(\frac{\alpha_{i_{1}}}{\alpha_{1}}, \frac{\alpha_{i_{2}}}{\alpha_{1}}, \frac{v_{i_{1}}}{v_{1}}, \frac{v_{j}}{v_{1}}\right) \cdot \alpha_{1} v_{1}+\frac{O P T(\mathbf{v})}{r} \geq \frac{O P T(\mathbf{v})}{r}
$$

and the proof follows.

It remains to prove inequality (9). We distinguish between three cases depending on the relative order of $j, i_{1}$, and $i_{2}$; in each of these cases, we further distinguish between two subcases. In each case, we exploit the structure of allocation $\pi$ to reason as follows. We consider a restriction of the original game (i.e., a different "restricted" game) by removing some advertisers from the original game and the slots they occupy in $\pi$. The particular advertisers to be removed are different in each case. We denote by $\pi^{\prime}$ the restriction of allocation $\pi$ to the advertisers and slots of the restricted game. We also use $\mathbf{v}^{\prime}$ to denote the valuation profile in the restricted game; so, $S W\left(\pi^{\prime}, \mathbf{v}^{\prime}\right)$ denotes the social welfare of $\pi^{\prime}$ in the restricted game. An important observation is that $\pi^{\prime}$ is a weakly feasible allocation in the restricted game since the 
weak feasibility conditions for $\pi^{\prime}$ are just a subset of the corresponding conditions for $\pi$ (for the original game). Furthermore, the restricted game has at most $n-1$ advertisers and, by the inductive hypothesis, we know that the inefficiency of $\pi^{\prime}$ is at most $r$. Then, inequality (9) follows using this fact and by carefully expressing the optimal social welfare in the new game.

Case I.1: $1<i_{1}<j<i_{2}$ and $\alpha_{j} \leq \alpha_{i_{2}} r$. Consider the restriction of the original game that consists of the advertisers different than $j, 1$, and $i_{1}$ and the slots different than $1, i_{1}$, and $i_{2}$. Let $\pi^{\prime}$ be the restriction of $\pi$ to the advertisers and slots of the new game and let $\mathbf{v}^{\prime}$ be the restriction of $\mathbf{v}$ to all advertisers besides $j, 1$ and $i_{1}$. Clearly, $\pi^{\prime}$ is weakly feasible for the new game since the weak feasibility conditions for $\pi^{\prime}$ are just a subset of the corresponding conditions for $\pi$ (for the original game). Also, note that the efficient allocation for the restricted game assigns advertiser $k$ to slot $k$ for $k=2, \ldots, i_{1}-1, i_{1}+1, \ldots, j-1, i_{2}+1, \ldots, n$ and advertiser $k+1$ to slot $k$ for $k=j, \ldots, i_{2}-1$. By the inductive hypothesis, we know that the inefficiency of $\pi^{\prime}$ is at most $r$. Hence, we can bound the social welfare of $\pi$ as

$$
\begin{aligned}
S W(\pi, \mathbf{v}) & =\alpha_{1} v_{j}+\alpha_{i_{1}} v_{1}+\alpha_{i_{2}} v_{i_{1}}+\sum_{k \notin\left\{1, i_{1}, i_{2}\right\}} \alpha_{k} v_{\pi(k)} \\
& =\alpha_{1} v_{j}+\alpha_{i_{1}} v_{1}+\alpha_{i_{2}} v_{i_{1}}+S W\left(\pi^{\prime}, \mathbf{v}^{\prime}\right) \\
& \geq \alpha_{1} v_{j}+\alpha_{i_{1}} v_{1}+\alpha_{i_{2}} v_{i_{1}}+\frac{1}{r}\left(\sum_{k=2}^{i_{1}-1} \alpha_{k} v_{k}+\sum_{k=i_{1}+1}^{j-1} \alpha_{k} v_{k}+\sum_{k=j}^{i_{2}-1} \alpha_{k} v_{k+1}+\sum_{k=i_{2}+1}^{n} \alpha_{k} v_{k}\right) \\
& \geq \alpha_{1} v_{j}+\alpha_{i_{1}} v_{1}+\alpha_{i_{2}} v_{i_{1}}+\frac{1}{r}\left(\sum_{k=2}^{i_{1}-1} \alpha_{k} v_{k}+\sum_{k=i_{1}+1}^{j-1} \alpha_{k} v_{k}+\sum_{k=j+1}^{i_{2}} \alpha_{k} v_{k}+\sum_{k=i_{2}+1}^{n} \alpha_{k} v_{k}\right) \\
& =\alpha_{1} v_{j}+\alpha_{i_{1}} v_{1}+\alpha_{i_{2}} v_{i_{1}}+\frac{1}{r}\left(\sum_{k=1}^{n} \alpha_{k} v_{k}-\alpha_{1} v_{1}-\alpha_{i_{1}} v_{i_{1}}-\alpha_{j} v_{j}\right) \\
& \geq \alpha_{1} v_{j}+\alpha_{i_{1}}\left(v_{1}-\frac{v_{i_{1}}}{r}\right)+\alpha_{i_{2}}\left(v_{i_{1}}-v_{j}\right)-\frac{\alpha_{1} v_{1}}{r}+\frac{O P T(\mathbf{v})}{r}
\end{aligned}
$$

and inequality (9) follows. The first inequality follows by the inductive hypothesis and the definition of the efficient allocation for the restricted game. The second inequality follows since $\alpha_{k} \geq \alpha_{k+1}$ for $k=$ $j, \ldots, i_{2}-1$. The last inequality follows since $\alpha_{j} \leq \alpha_{i_{2}} r$.

Case I.2: $1<i_{1}<j<i_{2}$ and $\alpha_{j}>\alpha_{i_{2}} r$. We use the restriction of the original game that consists of the advertisers different than $j$ and 1 and the slots different than 1 and $i_{1}$. Now, the efficient allocation for the restricted game assigns advertiser $k$ to slot $k$ for $k=2, \ldots, i_{1}-1, j+1, \ldots, n$ and advertiser $k-1$ to slot $k$ for $k=i_{1}+1, \ldots, j$. Using the inductive hypothesis for the restriction $\pi^{\prime}$ of $\pi$ to the restricted game, we 
can bound the social welfare of $\pi$ as

$$
\begin{aligned}
S W(\pi, \mathbf{v}) & =\alpha_{1} v_{j}+\alpha_{i_{1}} v_{1}+\sum_{k \notin\left\{1, i_{1}\right\}} \alpha_{k} v_{\pi(k)} \\
& =\alpha_{1} v_{j}+\alpha_{i_{1}} v_{1}+S W\left(\pi^{\prime}, \mathbf{v}^{\prime}\right) \\
& \geq \alpha_{1} v_{j}+\alpha_{i_{1}} v_{1}+\frac{1}{r}\left(\sum_{k=2}^{i_{1}-1} \alpha_{k} v_{k}+\sum_{k=i_{1}+1}^{j} \alpha_{k} v_{k-1}+\sum_{k=j+1}^{n} \alpha_{k} v_{k}\right) \\
& =\alpha_{1} v_{j}+\alpha_{i_{1}} v_{1}+\frac{1}{r}\left(\sum_{k=1}^{n} \alpha_{k} v_{k}-\alpha_{1} v_{1}-\alpha_{i_{1}} v_{i_{1}}+\sum_{k=i_{1}+1}^{j} \alpha_{k}\left(v_{k-1}-v_{k}\right)\right) \\
& \geq \alpha_{1} v_{j}+\alpha_{i_{1}} v_{1}+\frac{1}{r}\left(\sum_{k=1}^{n} \alpha_{k} v_{k}-\alpha_{1} v_{1}-\alpha_{i_{1}} v_{i_{1}}+\alpha_{j} \sum_{k=i_{1}+1}^{j}\left(v_{k-1}-v_{k}\right)\right) \\
& =\alpha_{1} v_{j}+\alpha_{i_{1}} v_{1}-\frac{1}{r}\left(\alpha_{1} v_{1}+\alpha_{i_{1}} v_{i_{1}}+\alpha_{j} v_{j}-\alpha_{j} v_{i_{1}}\right)+\frac{O P T(\mathbf{v})}{r} \\
& >\alpha_{1} v_{j}+\alpha_{i_{1}}\left(v_{1}-\frac{v_{i_{1}}}{r}\right)+\alpha_{i_{2}}\left(v_{i_{1}}-v_{j}\right)-\frac{\alpha_{1} v_{1}}{r}+\frac{O P T(\mathbf{v})}{r}
\end{aligned}
$$

and inequality (9) follows. The first inequality follows by the inductive hypothesis and the definition of the efficient allocation for the restricted game. The second inequality follows since $\alpha_{k} \geq \alpha_{j}$ and $v_{k-1}-v_{k} \geq 0$ for $k=i_{1}+1, \ldots, j$. The last inequality follows since $\alpha_{j}>\alpha_{i_{2}} r$.

Case II.1: $1<i_{1}<i_{2}<j$ and $v_{i_{2}} \leq v_{j} r$. We use the restriction of the original game that consists of the advertisers different than $j, 1$, and $i_{1}$ and the slots different than $1, i_{1}$, and $i_{2}$. Now, the efficient allocation for the restricted game assigns advertiser $k$ to slot $k$ for $k=2, \ldots, i_{1}-1, i_{1}+1, \ldots, i_{2}-1, j+1, \ldots, n$ and advertiser $k-1$ to slot $k$ for $k=i_{2}+1, \ldots, j$. Using the inductive hypothesis for the restriction $\pi^{\prime}$ of $\pi$ to the restricted game, we can bound the social welfare of $\pi$ as

$$
\begin{aligned}
S W(\pi, \mathbf{v}) & =\alpha_{1} v_{j}+\alpha_{i_{1}} v_{1}+\alpha_{i_{2}} v_{i_{1}}+\sum_{k \notin\left\{1, i_{1}, i_{2}\right\}} \alpha_{k} v_{\pi(k)} \\
& =\alpha_{1} v_{j}+\alpha_{i_{1}} v_{1}+\alpha_{i_{2}} v_{i_{1}}+S W\left(\pi^{\prime}, \mathbf{v}^{\prime}\right) \\
& \geq \alpha_{1} v_{j}+\alpha_{i_{1}} v_{1}+\alpha_{i_{2}} v_{i_{1}}+\frac{1}{r}\left(\sum_{k=2}^{i_{1}-1} \alpha_{k} v_{k}+\sum_{k=i_{1}+1}^{i_{2}-1} \alpha_{k} v_{k}+\sum_{k=i_{2}+1}^{j} \alpha_{k} v_{k-1}+\sum_{k=j+1}^{n} \alpha_{k} v_{k}\right) \\
& \geq \alpha_{1} v_{j}+\alpha_{i_{1}} v_{1}+\alpha_{i_{2}} v_{i_{1}}+\frac{1}{r}\left(\sum_{k=2}^{i_{1}-1} \alpha_{k} v_{k}+\sum_{k=i_{1}+1}^{i_{2}-1} \alpha_{k} v_{k}+\sum_{k=i_{2}+1}^{j} \alpha_{k} v_{k}+\sum_{k=j+1}^{n} \alpha_{k} v_{k}\right) \\
& =\alpha_{1} v_{j}+\alpha_{i_{1}} v_{1}+\alpha_{i_{2}} v_{i_{1}}+\frac{1}{r}\left(\sum_{k=1}^{n} \alpha_{k} v_{k}-\alpha_{1} v_{1}-\alpha_{i_{1}} v_{i_{1}}-\alpha_{i_{2}} v_{i_{2}}\right) \\
& \geq \alpha_{1} v_{j}+\alpha_{i_{1}}\left(v_{1}-\frac{v_{i_{1}}}{r}\right)+\alpha_{i_{2}}\left(v_{i_{1}}-v_{j}\right)-\frac{\alpha_{1} v_{1}}{r}+\frac{O P T(\mathbf{v})}{r}
\end{aligned}
$$

and inequality (9) follows. The first inequality follows by the inductive hypothesis and the definition of the efficient allocation for the restricted game. The second inequality follows since $v_{k-1} \geq v_{k}$ for $k=$ $i_{2}+1, \ldots, j$. The last inequality follows since $v_{i_{2}} \leq v_{j} r$. 
Case II.2: $1<i_{1}<i_{2}<j$ and $v_{i_{2}}>v_{j} r$. We use the restriction of the original game that consists of the advertisers different than 1 and $i_{1}$ and the slots different than $i_{1}$ and $i_{2}$. Now, the efficient allocation for the restricted game assigns advertiser $k$ to slot $k$ for $k=i_{2}+1, \ldots, n$, advertiser $i_{1}+1$ to slot $i_{1}-1$, and advertiser $k+1$ to slot $k$ for $k=1, \ldots, i_{1}-2, i_{1}+1, \ldots, i_{2}-1$. Using the inductive hypothesis for the restriction $\pi^{\prime}$ of $\pi$ to the advertisers and slots of the restricted game, we can bound the social welfare of $\pi$ as

$$
\begin{aligned}
S W(\pi, \mathbf{v})= & \alpha_{i_{1}} v_{1}+\alpha_{i_{2}} v_{i_{1}}+\sum_{k \notin\left\{i_{1}, i_{2}\right\}} \alpha_{k} v_{\pi(k)} \\
= & \alpha_{i_{1}} v_{1}+\alpha_{i_{2}} v_{i_{1}}+S W\left(\pi^{\prime}, \mathbf{v}^{\prime}\right) \\
\geq & \alpha_{i_{1}} v_{1}+\alpha_{i_{2}} v_{i_{1}}+\frac{1}{r}\left(\sum_{k=1}^{i_{1}-2} \alpha_{k} v_{k+1}+\alpha_{i_{1}-1} v_{i_{1}+1}+\sum_{k=i_{1}+1}^{i_{2}-1} \alpha_{k} v_{k+1}+\sum_{k=i_{2}+1}^{n} \alpha_{k} v_{k}\right) \\
= & \alpha_{i_{1}} v_{1}+\alpha_{i_{2}} v_{i_{1}}+\frac{1}{r}\left(\sum_{k=1}^{n} \alpha_{k} v_{k}+\sum_{k=1}^{i_{1}-2}\left(\alpha_{k}-\alpha_{k+1}\right) v_{k+1}+\left(\alpha_{i_{1}-1}-\alpha_{i_{1}+1}\right) v_{i_{1}+1}\right. \\
& \left.+\sum_{k=i_{1}+1}^{i_{2}-1}\left(\alpha_{k}-\alpha_{k+1}\right) v_{k+1}-\alpha_{1} v_{1}-\alpha_{i_{1}} v_{i_{1}}\right) \\
\geq & \alpha_{i_{1}} v_{1}+\alpha_{i_{2}} v_{i_{1}}+\frac{1}{r}\left(\sum_{k=1}^{n} \alpha_{k} v_{k}+\sum_{k=1}^{i_{1}-2}\left(\alpha_{k}-\alpha_{k+1}\right) v_{i_{2}}+\left(\alpha_{i_{1}-1}-\alpha_{i_{1}+1}\right) v_{i_{2}}\right. \\
& \left.+\sum_{k=i_{1}+1}^{i_{2}-1}\left(\alpha_{k}-\alpha_{k+1}\right) v_{i_{2}}-\alpha_{1} v_{1}-\alpha_{i_{1}} v_{i_{1}}\right) \\
= & \alpha_{i_{1}} v_{1}+\alpha_{i_{2}} v_{i_{1}}-\frac{1}{r}\left(\alpha_{1} v_{1}+\alpha_{i_{1}} v_{i_{1}}+\alpha_{i_{2}} v_{i_{2}}-\alpha_{1} v_{i_{2}}\right)+\frac{O P T(\mathbf{v})}{r} \\
> & \alpha_{1} v_{j}+\alpha_{i_{1}}\left(v_{1}-\frac{v_{i_{1}}}{r}\right)+\alpha_{i_{2}}\left(v_{i_{1}}-v_{j}\right)-\frac{\alpha_{1} v_{1}}{r}+\frac{O P T(\mathbf{v})}{r}
\end{aligned}
$$

and inequality (9) follows. The first inequality follows by the inductive hypothesis and the definition of the efficient allocation for the restricted game. The second inequality follows since $\alpha_{k}-\alpha_{k+1} \geq 0$ and $v_{k+1} \geq v_{i_{2}}$ for $k=1, \ldots, i_{1}-2, i_{1}+1, \ldots, i_{2}-1$ and $\alpha_{i_{1}-1}-\alpha_{i_{1}+1} \geq 0$ and $v_{i_{1}+1} \geq v_{i_{2}}$. The last inequality follows since $v_{i_{2}}>v_{j} r$ and $\alpha_{1}>\alpha_{i_{2}}$.

Case III.1: $1<i_{2}<i_{1}<j$ and $v_{i_{2}} \leq v_{j} r$. We use the restriction of the original game that consists of the advertisers different than $j, i_{1}$, and 1 and the slots different than $1, i_{2}$, and $i_{1}$. Now, the efficient allocation for the restricted game assigns advertiser $k$ to slot $k$ for $k=2, \ldots, i_{2}-1, j+1, \ldots, n$ advertiser $i_{1}-1$ to slot $i_{1}+1$, and advertiser $k-1$ to slot $k$ for $k=i_{2}+1, \ldots, i_{1}-1, i_{1}+2, \ldots, j$. Using the inductive 
hypothesis for the restriction $\pi^{\prime}$ of $\pi$ to the restricted game, we can bound the social welfare of $\pi$ as

$$
\begin{aligned}
S W(\pi, \mathbf{v})= & \alpha_{1} v_{j}+\alpha_{i_{2}} v_{i_{1}}+\alpha_{i_{1}} v_{1}+\sum_{k \notin\left\{1, i_{2}, i_{1}\right\}} \alpha_{k} v_{\pi(k)} \\
= & \alpha_{1} v_{j}+\alpha_{i_{2}} v_{i_{1}}+\alpha_{i_{1}} v_{1}+S W\left(\pi^{\prime}, \mathbf{v}^{\prime}\right) \\
\geq & \alpha_{1} v_{j}+\alpha_{i_{2}} v_{i_{1}}+\alpha_{i_{1}} v_{1}+\frac{1}{r}\left(\sum_{k=2}^{i_{2}-1} \alpha_{k} v_{k}+\sum_{k=i_{2}+1}^{i_{1}-1} \alpha_{k} v_{k-1}+\alpha_{i_{1}+1} v_{i_{1}-1}\right. \\
& \left.+\sum_{k=i_{1}+2}^{j} \alpha_{k} v_{k-1}+\sum_{k=j+1}^{n} \alpha_{k} v_{k}\right) \\
\geq & \alpha_{1} v_{j}+\alpha_{i_{2}} v_{i_{1}}+\alpha_{i_{1}} v_{1}+\frac{1}{r}\left(\sum_{k=2}^{i_{2}-1} \alpha_{k} v_{k}+\sum_{k=i_{2}+1}^{i_{1}-1} \alpha_{k} v_{k}+\sum_{k=i_{1}+1}^{n} \alpha_{k} v_{k}\right) \\
= & \alpha_{1} v_{j}+\alpha_{i_{2}} v_{i_{1}}+\alpha_{i_{1}} v_{1}+\frac{1}{r}\left(\sum_{k=1}^{n} \alpha_{k} v_{k}-\alpha_{1} v_{1}-\alpha_{i_{2}} v_{i_{2}}-\alpha_{i_{1}} v_{i_{1}}\right) \\
\geq & \alpha_{1} v_{j}+\alpha_{i_{1}}\left(v_{1}-\frac{v_{i_{1}}}{r}\right)+\alpha_{i_{2}}\left(v_{i_{1}}-v_{j}\right)-\frac{\alpha_{1} v_{1}}{r}+\frac{O P T(\mathbf{v})}{r}
\end{aligned}
$$

and inequality (9) follows. The first inequality follows by the inductive hypothesis and the definition of the efficient allocation for the restricted game. The second inequality follows since $v_{k-1} \geq v_{k}$ for $k=$ $i_{2}+1, \ldots, i_{1}-1, i_{1}+2, \ldots, j$ and $v_{i_{1}-1} \geq v_{i_{1}+1}$. The last inequality follows since $v_{i_{2}} \leq v_{j} r$.

Case III.2: $1<i_{2}<i_{1}<j$ and $v_{i_{2}}>v_{j} r$. We use the restriction of the original game that consists of the advertisers different than $i_{1}$ and 1 and the slots different than $i_{2}$ and $i_{1}$. Now, the efficient allocation for the restricted game assigns advertiser $k$ to slot $k$ for $k=i_{2}+1, \ldots, i_{1}-1, i_{1}+1, \ldots, n$ and advertiser $k+1$ to slot $k$ for $k=1, \ldots, i_{2}-1$. Using the inductive hypothesis for the restriction $\pi^{\prime}$ of $\pi$ to the restricted game, we can bound the social welfare of $\pi$ as

$$
\begin{aligned}
S W(\pi, \mathbf{v}) & =\alpha_{i_{2}} v_{i_{1}}+\alpha_{i_{1}} v_{1}+\sum_{k \notin\left\{i_{2}, i_{1}\right\}} \alpha_{k} v_{\pi(k)} \\
& =\alpha_{i_{2}} v_{i_{1}}+\alpha_{i_{1}} v_{1}+S W\left(\pi^{\prime}, \mathbf{v}^{\prime}\right) \\
& \geq \alpha_{i_{2}} v_{i_{1}}+\alpha_{i_{1}} v_{1}+\frac{1}{r}\left(\sum_{k=1}^{i_{2}-1} \alpha_{k} v_{k+1}+\sum_{k=i_{2}+1}^{i_{1}-1} \alpha_{k} v_{k}+\sum_{k=i_{1}+1}^{n} \alpha_{k} v_{k}\right) \\
& =\alpha_{i_{2}} v_{i_{1}}+\alpha_{i_{1}} v_{1}+\frac{1}{r}\left(\sum_{k=1}^{n} \alpha_{k} v_{k}-\alpha_{1} v_{1}-\alpha_{i_{1}} v_{i_{1}}+\sum_{k=1}^{i_{2}-1}\left(\alpha_{k}-\alpha_{k+1}\right) v_{k+1}\right) \\
\geq & \alpha_{i_{2}} v_{i_{1}}+\alpha_{i_{1}} v_{1}+\frac{1}{r}\left(\sum_{k=1}^{n} \alpha_{k} v_{k}-\alpha_{1} v_{1}-\alpha_{i_{1}} v_{i_{1}}+\sum_{k=1}^{i_{2}-1}\left(\alpha_{k}-\alpha_{k+1}\right) v_{i_{2}}\right) \\
& =\alpha_{i_{2}} v_{i_{1}}+\alpha_{i_{1}} v_{1}-\frac{1}{r}\left(\alpha_{1} v_{1}+\alpha_{i_{1}} v_{i_{1}}+\alpha_{i_{2}} v_{i_{2}}-\alpha_{1} v_{i_{2}}\right)+\frac{O P T(\mathbf{v})}{r} \\
& >\alpha_{1} v_{j}+\alpha_{i_{1}}\left(v_{1}-\frac{v_{i_{1}}}{r}\right)+\alpha_{i_{2}}\left(v_{i_{1}}-v_{j}\right)-\frac{\alpha_{1} v_{1}}{r}+\frac{O P T(\mathbf{v})}{r}
\end{aligned}
$$


and inequality (9) follows. The first inequality follows by the inductive hypothesis and the definition of the efficient allocation for the restricted game. The second inequality follows since $\alpha_{k}-\alpha_{k+1} \geq 0$ and $v_{k+1} \geq v_{i_{2}}$ for $k=1, \ldots, i_{2}-1$. The last inequality follows since $v_{i_{2}}>v_{j} r$ and $\alpha_{1}>\alpha_{i_{2}}$.

The proof of Theorem 4.1 is complete.

\section{Improved Bounds for Learning Outcomes}

In this section, we focus on the full information game. For simplicity we assume that all quality factors $\gamma_{i}=1$, and assume that players are sorted so that $v_{1} \geq v_{2} \geq \cdots \geq v_{n}$ (all proofs extend to the case with general quality factors by considering effective values $\gamma_{i} v_{i}$ in place of valuations everywhere).

The main goal of this Appendix is to prove Theorem 5.2. Similarly to the proof of Theorem 3.1 in Appendix $\mathrm{A}$ for Bayes-Nash equilibria, the proof considers a player $i$ with valuation $v_{i}$, possible bids of the form $y v_{i}$, and uses the fact that the player has no-regret about such alternative bids. In the full information case, we can handle the player with top valuation separately, and will only use that this player 1 has no regret about bidding her actual valuation $v_{1}$. For any other player $i$, the proof is analogous to the proof of Theorem 3.1 in Appendix A However, we no longer have to consider separately the case when the player's optimal slot is 1 . This allows us to drop one requirement for the function $g$ in the definition A.2. We further simplify that definition by setting $\delta=\beta$ (we have verified that different values for $\delta$ do not yield any improvement). More formally, we will need the following definition.

Definition C.1 Let $\beta \in(0,1]$. A function $g:[0,1] \rightarrow \mathbb{R}_{+}$is called $\beta$-bounded if the following two properties hold:

$$
\begin{array}{ll}
\text { i) } & \int_{0}^{1} g(y) \mathrm{d} y \leq 1, \\
\text { ii) } & \int_{z}^{1}(1-y) g(y) \mathrm{d} y \geq \beta-(1+\beta) z, \quad \forall z \in[0,1] .
\end{array}
$$

The following lemma states the connection of the price of anarchy to the existence of $\beta$-bounded functions.

Lemma C.2 Let $\beta \in(0,1]$ be such that a $\beta$-bounded function exists. Then, the price of total anarchy of the Generalized Second Price auction in the full information setting is at most $1+1 / \beta$.

Proof. In the proof, we consider a GSP auction game with $n$ slots with click-through-rates $\alpha_{1} \geq \alpha_{2} \geq$ $\ldots \geq \alpha_{n} \geq 0$ and $n$ conservative players with valuations $v_{1}, v_{2}, \ldots, v_{n} \geq 0$. Let $\mathbf{b}$ denote the bids of the players at a coarse correlated equilibrium.

We begin by lower-bounding the expected utility of each player at a coarse correlated equilibrium. We first consider player 1 and her deviation to the bid $v_{1}$. Then, player 1 would always be allocated slot 1 and would pay the highest bid among the remaining players (which is at most $b_{\pi(1)}$ ) per click. By the definition of the coarse correlated equilibrium such a deviation does not increase her expected utility (as the player has no regret), i.e.,

$$
\mathbb{E}\left[u_{1}(\mathbf{b})\right] \geq \mathbb{E}\left[u_{1}\left(v_{1}, \mathbf{b}_{-1}\right)\right] \geq \mathbb{E}\left[\alpha_{1}\left(v_{1}-b_{\pi(1)}\right)\right] \geq \beta \alpha_{1} v_{1}-\beta \mathbb{E}\left[\alpha_{1} b_{\pi(1)}\right],
$$

where the last inequality follows since $v_{1} \geq b_{\pi(1)}$ and since $\beta \in(0,1]$. Now, consider the deviation of player $i$ to the deterministic bid $b_{i}^{\prime} \leq v_{i}$. Then, she would be assigned to slot $i$ or higher and would get 
utility at least $\alpha_{i}\left(v_{i}-b_{i}^{\prime}\right)$ when the $i$-th highest bid is smaller than $b_{i}^{\prime}$. Again, by the definition of the coarse correlated equilibrium such a deviation does not increase her expected utility, i.e.,

$$
\mathbb{E}\left[u_{i}(\mathbf{b})\right] \geq \mathbb{E}\left[u_{i}\left(b_{i}^{\prime}, \mathbf{b}_{-i}\right)\right] \geq \mathbb{E}\left[\alpha_{i}\left(v_{i}-b_{i}^{\prime}\right) \mathbb{1}\left\{b_{\pi(i)}<b_{i}^{\prime}\right\}\right] .
$$

Using the first property in Definition C.1 for $g$ as well as the last inequality (with $b_{i}^{\prime}=y v_{i}$ ), we have

$$
\begin{aligned}
\mathbb{E}\left[u_{i}(\mathbf{b})\right] & \geq \int_{0}^{1} g(y) \cdot \mathbb{E}\left[u_{i}(\mathbf{b})\right] \mathrm{d} y \\
& \geq \int_{0}^{1} g(y) \cdot \mathbb{E}\left[\alpha_{i}\left(v_{i}-y v_{i}\right) \mathbb{1}\left\{b_{\pi(i)}<y v_{i}\right\}\right] \mathrm{d} y \\
& =\mathbb{E}\left[\alpha_{i} v_{i} \int_{0}^{1}(1-y) g(y) \mathbb{1}\left\{b_{\pi(i)}<y v_{i}\right\} \mathrm{d} y\right] \\
& =\mathbb{E}\left[\alpha_{i} v_{i} \int_{b_{\pi(i)} / v_{i}}^{1}(1-y) g(y) \mathrm{d} y\right] .
\end{aligned}
$$

We now apply the second property of Definition C.1 for function $g$ to obtain

$$
\mathbb{E}\left[u_{i}(\mathbf{b})\right] \geq \mathbb{E}\left[\beta \alpha_{i} v_{i}-(1+\beta) \alpha_{i} b_{\pi(i)}\right]=\beta \alpha_{i} v_{i}-(1+\beta) \mathbb{E}\left[\alpha_{i} b_{\pi(i)}\right] .
$$

By summing over all players and using inequalities (10) and (11), we have

$$
\begin{aligned}
\sum_{i} \mathbb{E}\left[u_{i}(\mathbf{b})\right] & =\mathbb{E}\left[u_{1}(\mathbf{b})\right]+\sum_{i \geq 2} \mathbb{E}\left[u_{i}(\mathbf{b})\right] \\
& \geq \beta \sum_{i} \alpha_{i} v_{i}-(1+\beta) \sum_{i} \mathbb{E}\left[\alpha_{i} b_{\pi(i)}\right]+\mathbb{E}\left[\alpha_{1} b_{\pi(1)}\right] \\
& =\beta O P T(\mathbf{v})-(1+\beta) \sum_{i} \mathbb{E}\left[\alpha_{i} b_{\pi(i)}\right]+\mathbb{E}\left[\alpha_{1} b_{\pi(1)}\right] .
\end{aligned}
$$

Now, we use this last inequality in the same way we used inequality (5) in the proof of Lemma A.1. By the fact that the social welfare is the sum of the expected utilities of the players plus the total payments, we obtain

$$
\begin{aligned}
\mathbb{E}[S W(\pi(\mathbf{b}), \mathbf{v})] & =\mathbb{E}\left[\sum_{i} u_{i}(\mathbf{b})\right]+\mathbb{E}\left[\sum_{i} \alpha_{i} b_{\pi(i+1)}\right] \\
& \geq \beta O P T(\mathbf{v})-(1+\beta) \sum_{i} \mathbb{E}\left[\alpha_{i} b_{\pi(i)}\right]+\mathbb{E}\left[\alpha_{1} b_{\pi(1)}\right]+\sum_{i \geq 2} \mathbb{E}\left[\alpha_{i} b_{\pi(i)}\right] \\
& =\beta O P T(\mathbf{v})-\beta \sum_{i} \mathbb{E}\left[\alpha_{i} b_{\pi(i)}\right] \\
& \geq \beta O P T(\mathbf{v})-\beta \mathbb{E}[S W(\pi(\mathbf{b}), \mathbf{v})],
\end{aligned}
$$

which implies that the price of total anarchy $O P T(\mathbf{v}) / \mathbb{E}[S W(\pi(\mathbf{b}), \mathbf{v})]$ is at most $1+1 / \beta$, as desired.

We are ready to complete the proof of Theorem 5.2 By Lemma C.2, it suffices to find a $\beta$-bounded function with $\beta$ as high as possible. Let $\lambda \approx 0.4328$ be the solution of the equation $1-\lambda+\ln (1-\lambda)=0$ and $g:[0,1] \rightarrow \mathbb{R}_{+}$be the function defined as follows:

$$
g(y)= \begin{cases}\frac{1}{(1-\lambda)(1-y)}, & y \in[0, \lambda] \\ 0, & y \in(\lambda, 1]\end{cases}
$$


We will show that $g$ is $\beta$-bounded for $\beta=\frac{\lambda}{1-\lambda}$; the upper bound of $1 / \lambda \approx 2.3102$ stated in Theorem 5.2 will then follow.

Indeed, by the definition of $\lambda$, we have

$$
\int_{0}^{1} g(y) \mathrm{d} y=\int_{0}^{\lambda} \frac{\mathrm{d} y}{(1-\lambda)(1-y)}=-\frac{\ln (1-\lambda)}{1-\lambda}=1 .
$$

Hence, $g$ satisfies the first property of Definition C.1. We also observe that

$$
\int_{z}^{1}(1-y) g(y) \mathrm{d} y=\int_{\min \{z, \lambda\}}^{\lambda} \frac{\mathrm{d} y}{1-\lambda} \geq \frac{\lambda-z}{1-\lambda}=\beta-(1+\beta) z,
$$

i.e., $g$ satisfies the second property of Definition C.1 as well.

\section{Irrational and Partially Rational Players}

In this section we consider the effect of partial rationality on the welfare generated by the GSP auction. We first consider a setting in which the players are not necessarily perfect utility optimizers, but rather can only be assumed to apply strategies that form an approximate equilibrium. We then study a setting in which some fraction of the players bid arbitrarily, without any rationality assumptions beyond avoiding the dominated strategy of overbidding (see Section 2.3. In both cases, we find that the social welfare guarantees of the GSP auction degrade continuously with the degree of irrationality present in the players.

\section{D.1 Approximate equilibria}

We will consider the social welfare generated by the GSP auction with uncertainty when players play only approximately utility-maximizing strategies. In Section 3, we assumed that rational players apply strategies at equilibrium. However, due to limits on rationality or indifference between small differences in utility, it may be the case that players converge only to an approximate equilibrium. We begin by defining this notion formally. Given a joint distribution $(\mathbf{F}, \mathbf{G})$ over types and quality factors, we say that strategy profile $\mathbf{b}$ is an $\epsilon$-Bayes-Nash equilibrium for distributions $\mathbf{F}, \mathbf{G}$ if, for all players $i$, all types $v_{i}$, and all alternative strategies $b_{i}{ }^{\prime}$,

$$
\mathbb{E}_{\mathbf{v}_{-i}, \gamma, \mathbf{b}}\left[u_{i}\left(b_{i}\left(v_{i}\right), \mathbf{b}_{-i}\left(\mathbf{v}_{-i}\right), \gamma\right) \mid v_{i}\right] \geq(1-\epsilon) \mathbb{E}_{\mathbf{v}_{-i}, \gamma, \mathbf{b}}\left[u_{i}\left(b_{i}^{\prime}\left(v_{i}\right), \mathbf{b}_{-i}\left(\mathbf{v}_{-i}\right), \gamma\right) \mid v_{i}\right] .
$$

Notice our choice of the multiplicative definition of approximate equilibria, justified by the fact that we have chosen not to scale values to lie in $[0,1]$.

We define the $\epsilon$-Bayes-Nash Price of Anarchy to be

$$
\sup _{\mathbf{F}, \mathbf{G}, \mathbf{b}(\cdot) \in \epsilon-B N E} \frac{\mathbb{E}_{\mathbf{v}, \gamma}[O P T(\mathbf{v}, \gamma)]}{\mathbb{E}_{\mathbf{v}, \gamma, \mathbf{b}(\mathbf{v})}[S W(\pi(\mathbf{b}(\mathbf{v}), \gamma), \mathbf{v}, \gamma)]}
$$

where $\epsilon$-BNE is the set of all $\epsilon$-Bayes-Nash equilibria.

We now claim that our bound for social welfare at (non-approximate) equilibrium degrades continuously as we relax the degree to which a bidding strategy only approximates an equilibrium.

Theorem D.1 The $\epsilon$-Bayes-Nash price of anarchy of the Generalized Second Price auction is at most $1.2553+(1-\epsilon)^{-1} \cdot 1.6722$. 
The intuition behind Theorem D.1 is that the bound for exact equilibria obtained in Theorem 3.1 depends on the Bayes-Nash equilibrium condition in a continuous way. This continuity is captured by the semismoothness of the GSP auction (as well as by inequality (5), used to prove Theorem 3.1 in Appendix A). Indeed, the following Lemma follows by a trivial modification to the proof of Lemma A.1.

Lemma D.2 Assume that for every GSP auction game there is a bidding profile $\mathbf{b}^{\prime}$ and parameters $\beta, \delta>0$ such that inequality (5) holds for any strategy profile $\mathbf{b}$. Then the $\epsilon$-Bayes-Nash price of anarchy of the Generalized Second Price auction is at most $\frac{(1-\epsilon)^{-1}+\delta}{\beta}$.

It then follows immediately from Lemmas A.3 and A.4 (see Appendix A) that the $\epsilon$-Bayes-Nash price of anarchy of the GSP auction is at most $\frac{(1-\epsilon)^{-1}+0.7507}{0.7507 \cdot 0.7966} \approx 1.2553+(1-\epsilon)^{-1} \cdot 1.6722$.

\section{D.2 Irrational players}

We now consider a setting in which, of the $n$ advertisers who bid in the GSP auction, some subset of them are "irrational" and cannot be assumed to apply strategies at equilibrium. We still think of the irrational advertisers as being true players in the GSP auction, with valuations and quality scores. The irrational advertisers simply may not apply rational bidding strategies; for example, they may not have experience with the GSP auction, or not know about historical bidding patterns.

Our setting will be an extension of the GSP auction with uncertainty. We will first provide some definitions. Given valuations $\mathbf{v}$, quality scores $\gamma$, an outcome $\pi$, and a set $S$ of players, the social welfare restricted to set $S$ is $S W_{S}(\pi, \mathbf{v}, \gamma)=\sum_{i \in S} \alpha_{\pi^{-1}(i)} \gamma_{i} v_{i}$, the total value of the outcome $\pi$ for the advertisers in $S$. The optimal social welfare restricted to $S$ is $O P T_{S}(\mathbf{v}, \gamma)=\max _{\pi} S W_{S}(\pi, \mathbf{v}, \gamma)$.

Given a joint distribution $(\mathbf{F}, \mathbf{G})$ over types and quality factors, and a set $S$ of players, we say that strategy profile $\mathbf{b}$ is an $S$-Bayes-Nash equilibrium for distributions $\mathbf{F}, \mathbf{G}$ if, for all players $i \in S$, all types $v_{i}$, and all alternative strategies $b_{i}{ }^{\prime}$,

$$
\mathbb{E}_{\mathbf{v}_{-i}, \gamma, \mathbf{b}}\left[u_{i}\left(b_{i}\left(v_{i}\right), \mathbf{b}_{-i}\left(\mathbf{v}_{-i}\right), \gamma\right) \mid v_{i}\right] \geq \mathbb{E}_{\mathbf{v}_{-i}, \gamma, \mathbf{b}}\left[u_{i}\left(b_{i}^{\prime}\left(v_{i}\right), \mathbf{b}_{-i}\left(\mathbf{v}_{-i}\right), \gamma\right) \mid v_{i}\right] .
$$

That is, no player in $S$ can improve her utility by modifying her bid, but no such restriction is imposed upon the players outside $S$.

We will show that, for each set $S$ of players, the total expected social welfare obtained by GSP at an $S$-Bayes-Nash equilibrium is a good approximation to $\mathbb{E}\left[O P T_{S}(\mathbf{v})\right]$. We can interpret this result as stating that the addition of irrational players does not significantly degrade the social welfare that would have been generated had they not participated. Note that we cannot hope to always obtain a good approximation to $\mathbb{E}[O P T(\mathbf{v})]$ (the optimal social welfare of all advertisers) at all $S$-Bayes-Nash equilibria; for example, it may be that the valuations of the players outside $S$ are very large, but they choose (irrationally) to bid 0 .

We note that our no-overbidding assumption (Section 2.3) continues to apply to all players, not only to the players in $S$. In other words, we require that $b_{i}\left(v_{i}\right) \leq v_{i}$ for all $i \notin S$ and all $v_{i}$. We feel this is a natural restriction to impose even on "irrational" advertisers, as overbidding is an easily-avoided dominated strategy. Moreover, it is arguable that inexperienced advertisers would bid conservatively, and not risk a large payment with no gain 6

\footnotetext{
${ }^{6}$ This relies on the simplifying assumption that all advertisers have knowledge of their own private valuations. Admittedly, this requires a certain level of sophistication and may be difficult to attain in practice. Our argument is thus limited to imperfect strategy choice given perfect knowledge of types. It remains open to extend this analysis to players who may misunderstand their own valuations.
} 
Formally, given a non-empty subset $S$ of advertisers, we define the $S$-Bayes-Nash Price of Anarchy to be

$$
\sup _{\mathbf{F}, \mathbf{G}, \mathbf{b}(\cdot) \in S-B N E} \frac{\mathbb{E}_{\mathbf{v}, \gamma}\left[O P T_{S}(\mathbf{v}, \gamma)\right]}{\mathbb{E}_{\mathbf{v}, \gamma, \mathbf{b}(\mathbf{v})}[S W(\pi(\mathbf{b}(\mathbf{v}), \gamma), \mathbf{v}, \gamma)]}
$$

where $S$-BNE is the set of all $S$-Bayes-Nash equilibria.

Our main result is the following extension of Theorem 3.1

Theorem D.3 For any non-empty subset $S$ of rational advertisers, the S-Bayes-Nash price of anarchy of the Generalized Second Price auction is at most 2.927.

In order to prove this theorem, we need an inequality similar to inequality (5) in Section A, In particular, for every bid profile $\mathbf{b}$, there exists a bid profile $\mathbf{b}^{\prime}$ defined on the rational advertisers such that

$$
\sum_{i \in S} \mathbb{E}\left[u_{i}\left(b_{i}^{\prime}\left(v_{i}\right), \mathbf{b}_{-i}, \gamma\right)\right] \geq \beta \mathbb{E}\left[O P T_{S}(\mathbf{v}, \gamma)\right]-(1+\delta) \sum_{i \in S} \mathbb{E}\left[\alpha_{\nu(i)} \gamma_{\pi(\nu(i))} b_{\pi(\nu(i))}\right]+\mathbb{E}\left[\alpha_{1} \gamma_{\pi(1)} b_{\pi(1)}\right]
$$

We can prove inequality (12) by following the same steps as in the proof of Lemma A.3 and by considering the utilities of the rational players at their most profitable deviation. Here, $\nu(i)$ should be interpreted as the slot the rational advertiser $i$ occupies in the efficient allocation restricted to $S$ and $\pi(j)$ is the advertiser that occupies the $j$-th slot in allocation $\pi$ (this advertiser can be rational or irrational). Similarly, $\pi^{i}(j)$ is the player with the $j$-th highest effective bid among all advertisers besides the rational advertiser $i$.

All the arguments hold in this case as well. However, there is a minor point that should be justified. Observe that in order to obtain inequalities (7) and (8), we used the fact that the $j$-th highest effective bid (excluding advertiser $i$ ) is not larger that the effective value of advertiser $i$ when $\nu(i)=j$, i.e., when slot $j$ is allocated to advertiser $i$ in the efficient allocation restricted to $S$. When adapting the proof to the case of rational and irrational players, it may be the case that $\nu(i)=j$ when the rational advertiser $i$ has valuation $v_{i}=x$ but $\gamma_{\pi^{i}(j)} b_{\pi^{i}(j)}>\gamma_{i} x$. This may be due to the fact that player $\pi^{i}(j)$ is one of the irrational players. Fortunately, both inequalities (7) and (8) are obviously true in this case as well. Observe that $\beta \leq \delta$ (otherwise, the second property of Definition A.2 would not hold for $z=1$ ) and the right-hand side of both inequalities is non-positive. The changes in the rest of the proof of Lemma A.3 are minor.

Then, Theorem D.3 follows by the next lemma that exploits inequality (12) and using the same values for $\beta$ and $\delta$ that we used in Section A

Lemma D.4 Assume that for every GSP auction game with a non-empty set $S$ of rational players there is a bidding profile $\mathbf{b}^{\prime}$ for the players in $S$ and parameters $\beta, \delta>0$ such that inequality (12) holds for any strategy profile $\mathbf{b}$. Then, the S-Bayes-Nash price of anarchy of the Generalized Second Price auction is at $\operatorname{most} \frac{1+\delta}{\beta}$.

Proof. Consider an $S$-Bayes-Nash equilibrium $\mathbf{b}$. Define $\mathbf{b}^{\prime}$ as in inequality $(12)$ and observe that $\mathbb{E}\left[u_{i}(\mathbf{b}, \gamma)\right] \geq$ $\mathbb{E}\left[u_{i}\left(b_{i}^{\prime}\left(v_{i}\right), \mathbf{b}_{-i}, \gamma\right)\right]$ for every player $i \in S$. We use this inequality and the fact that the social welfare is at 
least the sum of the expected utilities of the rational advertisers plus the total payments to get

$$
\begin{aligned}
& \mathbb{E}[S W(\pi(\mathbf{b}(\mathbf{v}), \gamma), \mathbf{v}, \gamma)] \geq \sum_{i \in S} \mathbb{E}\left[u_{i}(\mathbf{b}, \gamma)\right]+\sum_{i} \mathbb{E}\left[\alpha_{i} \gamma_{\pi(i+1)} b_{\pi(i+1)}\right] \\
& \quad \geq \sum_{i \in S} \mathbb{E}\left[u_{i}\left(b_{i}^{\prime}\left(v_{i}\right), \mathbf{b}_{-i}, \gamma\right)\right]+\sum_{i \geq 2} \mathbb{E}\left[\alpha_{i} \gamma_{\pi(i)} b_{\pi(i)}\right] \\
& \quad \geq \beta \mathbb{E}\left[O P T_{S}(\mathbf{v}, \gamma)\right]-(1+\delta) \sum_{i \in S} \mathbb{E}\left[\alpha_{\nu(i)} \gamma_{\pi(\nu(i))} b_{\pi(\nu(i))}\right]+\mathbb{E}\left[\alpha_{1} \gamma_{\pi(1)} b_{\pi(1)}\right]+\sum_{i \geq 2} \mathbb{E}\left[\alpha_{i} \gamma_{\pi(i)} b_{\pi(i)}\right] \\
& \quad \geq \beta \mathbb{E}\left[O P T_{S}(\mathbf{v}, \gamma)\right]-(1+\delta) \sum_{i} \mathbb{E}\left[\alpha_{i} \gamma_{\pi(i)} b_{\pi(i)}\right]+\sum_{i} \mathbb{E}\left[\alpha_{i} \gamma_{\pi(i)} b_{\pi(i)}\right] \\
& \quad=\beta \mathbb{E}\left[O P T_{S}(\mathbf{v}, \gamma)\right]-\delta \sum_{i} \mathbb{E}\left[\alpha_{i} \gamma_{\pi(i)} b_{\pi(i)}\right] \\
& \quad \geq \beta \mathbb{E}\left[O P T_{S}(\mathbf{v}, \gamma)\right]-\delta \mathbb{E}[S W(\pi(\mathbf{b}(\mathbf{v}), \gamma), \mathbf{v}, \gamma)],
\end{aligned}
$$

which implies that the $S$-Bayes-Nash price of anarchy is at most $\frac{1+\delta}{\beta}$, as desired. 